\title{
The YOHKOH survey of partially occulted flares in hard X-rays
}

\author{
M. Tomczak \\ Astronomical Institute, University of Wrocław, ul. Kopernika 11, 51-622 Wrocław, Poland \\ e-mail: tomczak@astro.uni.wroc.pl
}

Received 27 January 2009 / Accepted 22 April 2009

\begin{abstract}
Context. Modern solar X-ray imagers do not completely resolve the problem of deriving detailed diagnostics of faint hard X-ray sources in the presence of stronger ones. This is the case for the impulsive phase of solar flares in which footpoint sources are usually stronger than loop-top ones.

Aims. Flares that are partially occulted by the solar limb provide the most hopeful source of knowledge about hard X-ray loop-top sources. This work attempts to fill the gap between the published survey of partially occulted flares observed by RHESSI (Krucker \& Lin 2008, ApJ, 673, 1181) and the extensive Yohkoh database.

Methods. Among the 1286 flares in the Yohkoh Hard X-ray Telescope Flare Catalogue (Sato et al. 2006, Sol. Phys., 236, 351), for which the hard X-ray images were presented, we identified 98 events that occurred behind the solar limb. We investigated their hard $\mathrm{X}$-ray spectra and spatial structure.

Results. In most cases, we found that the hard X-ray spectrum of partially occulted flares consists of two components, non-thermal and thermal, which are cospatial to within 4 arcsec. For rest events, the components are separated, the non-thermal component clearly appearing to be situated higher. The photon energy spectra of the partially occulted flares are systematically steeper than spectra of the non-occulted flares. We can explain this difference as a consequence of intrinsically dissimilar conditions in coronal parts of flares, in comparison with the footpoints that usually dominate the hard X-ray emission of disk flares. At least two reasons for the difference should be taken into consideration: (1) stronger contamination of hard X-rays by emission from thermal plasma; and (2) different mechanisms in which non-thermal electrons radiate their energy. For events unbiased by the thermal component, the difference, $\Delta \gamma=\bar{\gamma}_{\mathrm{LT}}-\bar{\gamma}_{\mathrm{FP}}$, equals 1.5. We found a lack of correlation between the altitude of flares and the hard X-ray power-law index $\gamma$.

Conclusions. A schematic picture, in which a thin-target mechanism is responsible for the hard X-ray emission of loop-top sources and a thick-target mechanism for emission by footpoint sources, is modified by the presence of some coronal thick-target sources. Some of these sources exhibit evidence of magnetic trapping. For the characteristics of flares is conclusive the local magnetic configuration in which they occur.
\end{abstract}

Key words. Sun: corona - Sun: flares - Sun: particle emission - Sun: X-rays, gamma rays

\section{Introduction}

It has been commonly accepted that solar flares are caused by the reconnection of magnetic field lines in the corona. In this process, energy originally stored in the magnetic field is redistributed into plasma heating, wave generation, and particle acceleration. However, details of the reconnection process as well as general rules about energy partition are subjects of extensive debate. Any progress strongly depends on carefully planned observations and their interpretation.

Hard X-ray observations offer good insight into further evaluation of particles accelerated in the reconnection process. The propagation of particles operates under the guidance of the magnetic lines. Their bundles converge at the entrance into the lower part of the solar atmosphere, where the density of the ambient plasma increases steeply. Electrons accelerated in the corona are stopped there, emitting intense hard X-radiation via electronion bremsstrahlung. This mechanism, known as the thick-target model (Brown 1971), works so efficiently that footpoint hard $\mathrm{X}$-ray sources usually strongly dominate the spatial distribution of flare emission in this energy range.

It is difficult to observe the particle acceleration without effects introduced by the propagation of particles because in the less-dense corona, hard X-radiation is emitted less efficiently in the thin-target model (Brown 1971; Lin 1974). In many cases, the coronal hard X-ray sources can be seen as the effect of magnetic convergence (Tomczak \& Ciborski 2007) or ultra-dense thick-target environment (Kosugi et al. 1994; Veronig \& Brown 2004).

Coronal hard X-ray sources can be easily distinguished when a flare occurs behind the solar limb but close enough to record emission from the higher part of the magnetic structure. In the case of a partially occulted flare, the solar disk works like a rough imager that stops the emission of usually brighter footpoint sources. Such a configuration had been used routinely for investigation of coronal hard X-ray sources before hard X-ray imaging detectors began to operate (Zirin et al. 1969; Frost \& Dennis 1971; Hudson 1978; Hudson et al. 1982; Kane et al. 1982).

A first statistical attempt to analyze partially occulted flares was performed using data from the UCSD experiment onboard the OSO-7 satellite (Roy \& Datlowe 1975; McKenzie 1975). In fifteen months of OSO-7 operation, from among $601 \mathrm{X}$-ray bursts above $10^{3}$ photon $\mathrm{cm}^{-2} \mathrm{~s}^{-1} \mathrm{keV}^{-1}$ in energy channel extending over $5.1-6.6 \mathrm{keV}, 54$ bursts were unaccompanied by $\mathrm{H} \alpha$ flares. Since a cinema flare patrol was in progress during those bursts, they probably occurred behind the solar limb. 
From the group of partially occulted flares, McKenzie (1975) chose eight major soft X-ray events and found that all had significant hard X-ray emission in the $30-44 \mathrm{keV}$ range. Roy \& Datlowe (1975) analyzed all available hard X-ray spectra of partially occulted flares. They found that 25 of 37 bursts had a nonthermal component. The average value of the spectral indices at peak $20 \mathrm{keV}$ flux for these 25 over-the-limb events was 4.6, whereas for 59 center events $\left(0^{\circ}<\theta<60^{\circ}\right)$ it was 3.8.

The main conclusion of the above mentioned papers from the pre-imaging era was that hard X-ray emission was not concentrated close to the solar surface but took place in extended regions in the corona.

Modern hard X-ray telescopes onboard the Yohkoh (Hard X-ray Telescope) and Reuven Ramaty High-Energy Solar Spectroscopic Imager (RHESSI) satellites have opened a new perspective in investigating coronal hard X-ray sources. Many important discoveries have been made (Krucker et al. 2008a) by including the most famous - the discovery of the presence of above the loop-top sources in flares (Masuda et al. 1994). However, in many cases the low dynamical range of a hard $\mathrm{X}$-ray telescope creates problems for even a qualitative analysis of images, since detailed diagnostics of faint hard X-ray sources become difficult to measure in the presence of stronger ones. Alexander \& Metcalf (1997) demonstrated that weak sources can be suppressed during the image reconstruction and mimic the appearance of stronger neighbors. This is indeed true for the impulsive phase of a typical flare when we observe strong footpoint and weak loop-top sources simultaneously.

To avoid this complication, partially occulted flares have been studied. From the Yohkoh database, one or a few examples of behind-the-limb flares have previously been selected many times. This specific configuration was usually used to derive joint diagnostics from hard and soft X-ray images by including full-Sun soft X-ray spectra recorded by the Bragg Crystal Spectrometer. In this way, soft X-ray bright loop-top kernels (Khan et al. 1995; Mariska et al. 1996; Sterling et al. 1996; Mariska \& Doschek 1997; Ohyama \& Shibata 1997) as well as flare-associated X-ray plasma ejections (Ohyama \& Shibata 1997; Tomczak 2004, 2005) were investigated.

Mariska \& McTiernan (1999) compared the hard and soft X-ray characteristics of 28 partially occulted and 17 nonocculted limb flares observed by Yohkoh between 1991 and 1996. For most observational characteristics, partially occulted flares were found to be indistinguishable from non-occulted ones. Exceptions were found for hard X-ray spectra averaged over entire events, which exhibited higher values of the powerlaw index $\gamma$ in the partially occulted flares.

Tomczak (2001) investigated the Yohkoh X-ray images of 14 behind-the-limb flares. He reported their complex variability in hard X-ray flux which could be reproduced by two separate components: gradual lower-energy backgrounds lasting several minutes and quasi-periodic higher-energy impulses lasting typically 5-30 s. Impulses were too weak for imaging but gradual components enables an easy identification in hard X-ray images. The hard X-ray sources were usually cospatial with soft X-ray kernels, but sometimes had no distinct counterparts in soft X-rays. The appearance of a new gradual component in the hard X-ray light curve was always associated with the presence of an additional hard X-ray source.

Observations of the partially occulted flares were analyzed with RHESSI data (Krucker et al. 2007a; Li \& Gan 2007; Liu et al. 2008), and either Hinode (Krucker et al. 2007b) or Solar-Terrestrial Relations Observatory, STeReO (Krucker et al. 2009) data. Krucker \& Lin (2008) prepared a survey of partially occulted flares observed by RHESSI. They detected 55 of these events between 2002 February and 2004 August. Two different components of coronal hard X-ray emission were detected in 50 flares: (1) a thermal component with a gradual time profile; and (2) a non-thermal (power-law spectra with indices $\gamma$ mostly between 4 and 7) component exhibiting more rapid time variations. Both components were usually cospatial within $\sim 2 \times 10^{3} \mathrm{~km}$ with only a few exceptions.

The interpretation of the extensive Yohkoh database would benefit from a survey of partially occulted flares, which this paper attempts to complete using the Hard X-ray Telescope Flare Catalogue. The development and structure of this catalogue are described in Sect. 2. The criteria of selection of flares enclosed in the survey are given in Sect. 3. In Sect. 4, a statistical approach to some characteristics describing partially occulted flares is given and the results are compared to their counterparts obtained for non-occulted flares from the catalogue. In Sect.5, the results are discussed and compared to the reports of other authors. In Sect. 6, short descriptions of some interesting groups of events in the catalogue are given. Our main conclusions are summarized in Sect. 7.

\section{Development and structure of the YOHKOH HXT Flare Catalogue}

The Japanese solar satellite Yohkoh operated during the years 1991-2001 providing a huge amount of excellent data. The Hard X-ray Telescope, HXT, (Kosugi et al. 1991) was a Fourier synthesis imager observing the whole Sun. It consisted of 64 independent subcollimators measuring spatially modulated intensities in four energy bands (L: 14-23 keV, M1: 23-33 keV, M2: $33-53 \mathrm{keV}$, and $\mathrm{H}: 53-93 \mathrm{keV})$. During the flare, the intensities were integrated, in each energy band, over $0.5 \mathrm{~s}$. Some reconstruction routines (Maximum Entropy Method, Pixons) that allow us to obtain hard X-ray images with an angular resolution of up to 5 arcsec are available.

Among the four scientific instruments onboard Yohkoh, the observations made by the HXT were organized in the most friendly way since the early years of the mission. The members of the HXT team prepared five versions of a catalogue by collecting basic information about flares observed by the telescope (see Table 1). The first two versions of the catalogue (Kosugi et al. 1993, 1995) contained 672 and 1007 records each. For some flares, the $10 \mathrm{~min}$ hard X-ray light curves in four energy bands were provided. In the 3rd version of the catalogue (Sato et al. 1998) containing 1264 records, example hard X-ray images were added. The last two versions of the catalogue contain the entire mission database with 3112 records and some supplementary data from other Yohkoh instruments. In the 4th version (Sato et al. 2003), the example soft X-ray images provided by the Soft $\mathrm{X}$-ray Telescope (SXT) were introduced. In the 5th version (Sato et al. 2006), light curves and spectra provided by the Wide Band Spectrometer (WBS) were introduced.

In summary, the 5 th version of the catalogue provides a short description of the $3090^{1}$ flares that produced at least 3 counts s $\mathrm{s}^{-1}$ per subcollimator $\left(\mathrm{SC}^{-1}\right)$ in channel $\mathrm{L}$. The description contains date, time, peak counts in both four HXT and four WBS energy bands, GOES class, $\mathrm{H} \alpha$ position, and NOAA active region number. For 1286 flares, example images in particular energy

\footnotetext{
1 Note that the total number of flares is slightly lower than the total number of records because more than one record is assigned to some flares.
} 
Table 1. Development of the YOHKOH HXT Flare Catalogue.

\begin{tabular}{ccccccccc}
\hline \hline Version & \multirow{2}{*}{ References } & Latest & & \multicolumn{5}{c}{ Contents } \\
\cline { 5 - 9 } & event & Records & $\begin{array}{c}\text { HXT } \\
\text { (lc) })^{a}\end{array}$ & $\begin{array}{c}\text { HXT } \\
(\mathrm{i})^{a}\end{array}$ & GOES & SXT & WBS \\
\hline 1 & Kosugi et al. (1993) & $1992 / 12$ & 672 & + & - & + & - & - \\
2 & Kosugi et al. (1995) & $1994 / 12$ & 1007 & + & - & + & - & - \\
3 & Sato et al. (1998) & $1998 / 08$ & 1264 & + & + & + & - & - \\
4 & Sato et al. (2003) & $2001 / 12$ & 3112 & + & + & + & + & - \\
5 & Sato et al. (2006) & $2001 / 12$ & 3112 & + & + & + & + & + \\
\hline
\end{tabular}

${ }^{a}$ lc - light curves; i - images.
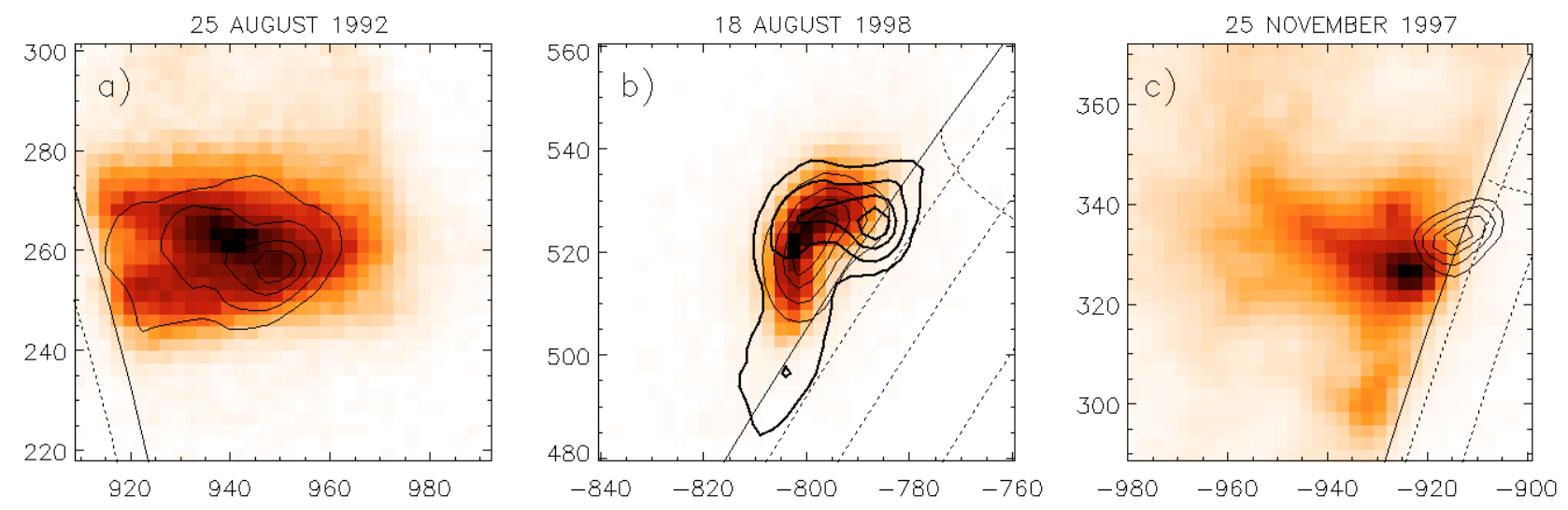

Fig. 1. Three examples of events that were considered during the preparation of the survey of partially occulted flares. Soft X-ray images (halftones) were obtained by the SXT in the Be119 filter, thin contours $\left(10,25,50\right.$, and $75 \%$ of $\left.I_{\max }\right)$ representing hard X-ray emission in energy band L (14-23 keV). Solid line shows solar limb and dashed lines represent heliospheric coordinates on the solar disk. a) Event included in the survey showing a typical coronal source. b) Event excluded from the survey because of footpoint sources seen in the energy band M2 (33-53 keV) - thick contours. c) Event included in the survey but excluded from statistical considerations due to possibly footpoint contamination (estimated longitude $\theta=\mathrm{E} 91.6 \pm 0.6)$.

bands, basically for a peak time period, are enclosed. The criterion for inclusion of an image in the catalogue was a count rate of roughly 10 counts s ${ }^{-1} \mathrm{SC}^{-1}$ above the background. The HXT images are superimposed on a SXT image when available. For the same 1286 flares four HXT light curves are presented. The catalogue also contains WBS light curves of 2736 flares. Example WBS spectra of 369 flares for energies up to $1 \mathrm{MeV}$ and spectra of 12 flares for energies up to $10 \mathrm{MeV}$ are added for a peak time period.

The last version of the catalogue still needs some improvements and corrections. For example, for the years 1997-2001 an identification of GOES class, $\mathrm{H} \alpha$ position, and NOAA active region number was made only for a minor part of events. The poor quality of some HXT images implies that their positions had been determined incorrectly, a conclusion that would agree with the observed disagreement with the $\mathrm{H} \alpha$ position of a flare.

\section{Criteria of selection}

In the first step, all hard X-ray images available in the catalogue were inspected. As a result, the preliminary list of 170 events, located close to the solar limb, was established. Each event from the preliminary list was then carefully verified using original files of data. For flares that occur behind the solar limb, the footpoint hard X-ray sources should not be detected. Therefore, every flare from the list, for which a centroid of any hard $\mathrm{X}$-ray sources was located within the solar disk was rejected. If soft X-ray images of the flare were available, a decision about rejection was made by using these data. For flares that occur behind the solar limb, the solar limb appears to sharply intersect the soft X-ray emission. The counterparts of footpoint hard Xray sources are also soft X-ray brightenings (Hudson et al. 1994; Tomczak 1997). Thus, for events in our survey, the soft X-ray brightenings should not be detected. If images of the flare taken during the time interval chosen in the catalogue did not allow us to decide, we used images for other time interval. Three examples of flares considered during the preparation of the survey are presented in Fig. 1.

Finally, we identified 98 flares that observations strongly suggested were behind the solar limb. The list of them is presented in Table 2. How far behind the solar limb are they located? The accurate knowledge of heliographic longitude, $\theta$, is crucial to a correct measurement of altitude above the photosphere, $h$, one of the most important parameters in our discussion below. However, before the launch of the $S T e R e O$ satellites, only a limited quality of estimation of the longitude was possible.

We estimate this parameter in two different ways. Our first method employed beyond-the-limb extrapolation of a straight line fitted to the plot of longitude versus time for all $\mathrm{H} \alpha$ flares observed in the proper active region during its passage across the solar disk (Roy \& Datlowe 1975). In this approach, observations collected in the Solar-Geophysical Data (SGD) were used and an example of the longitude extrapolation for the flare No. 23070 in the Catalogue from the NOAA AR 9087 is given in Fig. 2. In our second method we calculated the flare position on the basis of the time of central meridian passage (CMP) taken from the SGD for the proper active region. The formula describing the 


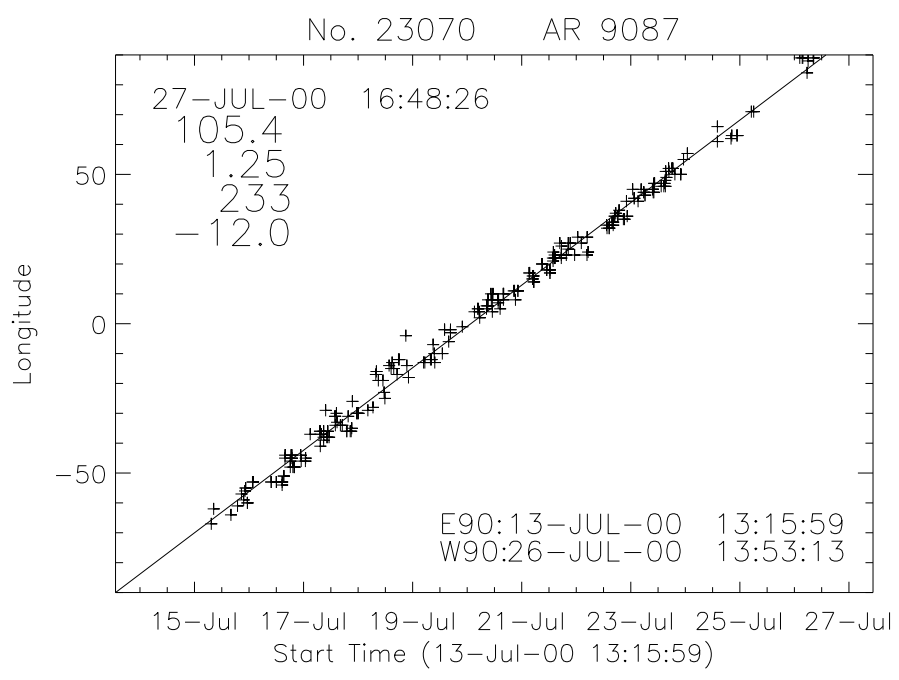

Fig. 2. Example of the longitude extrapolation. Description on the plot shows: time of event, value of $\theta$ and its uncertainty, number of points, average value of the latitude (top, left), and times of limb occurrences (bottom, right).

solar differentional rotation given by Newton \& Nunn (1951) for large recurrent sunspots, corrected by Ward (1966), was used:

$\theta=13.45-3.0 \sin ^{2} \phi \quad \operatorname{deg}_{\text {day }}{ }^{-1}$,

where $\phi$ is the region latitude.

Both methods of estimating $\theta$ work well only for specific circumstances. The first shows the position of a weighted centroid of positions in which flares occurred in the active region during its passage across the solar disk. The second basically provides the position of the central part of an active region. Thus, if the investigated behind-the-limb flare occurred in an alternative place in the active region, each method may be affected some additional (systematic) error. This is why the derived values of $\theta$ were sometimes lower than $90^{\circ}$, although the available X-ray images provided strong indication that their positions beyond the solar limb were.

The values of $\theta$ given in Col. (6) of Table 2 were basically obtained by the first method, except for when the $\theta-t$ plot consisted of only a few points or the second method provided a more realistic solution. Uncertainties given in Col. (7) of Table 2 provide a measure of the scatter in the source data taken from the SGD. The results are summarized in Fig. 3, where the latitude is inferred directly from the hard X-ray image. The measured values of the longitude range between slightly below $90^{\circ}$ (a consequence of systematic errors) and $26^{\circ} .9 \pm 1.8$ beyond the solar limb (event No. 26060). For event No. 15640 alone, we were unable to estimate the longitude.

At first glance, it seems that in our survey some partially occulted flares have been missed. We identified only 98 events out of 1286 , i.e., $7.6 \%$ of the catalogue sources. Assuming a uniform distribution of flare longitude in the catalogue, one should expect that $4 / 22$, i.e., $18.2 \%$ of the flares occurred behind the solar limb between $0^{\circ}$ and $20^{\circ}$. We should recall, however, that the further the position of a flare beyond the solar limb, the larger is the occultation height. For $10^{\circ}$, hard X-ray emission should for example, occur at least about $\sim 1.1 \times 10^{4} \mathrm{~km}$ above the photosphere to be seen and for $20^{\circ}$ the minimal height increases to about $\sim 4.6 \times 10^{4} \mathrm{~km}$. This effect seriously decreases the population of partially occulted flares.

We estimated the corrected population of behind-the-limb flares by including the frequency of their occurrence in their

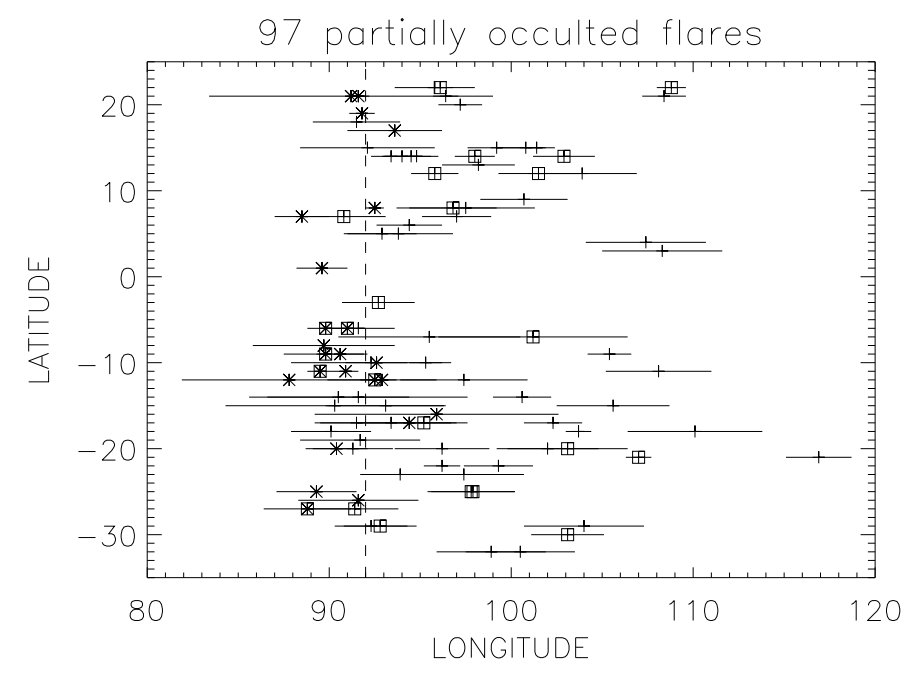

Fig. 3. Plot of heliographic coordinates (longitude versus latitude) for 97 flares from our survey. Events observed outside the maximum of hard X-rays are marked with boxes, events with possibly footpoint contamination are indicated by stars. The termination longitude for contamination $\left(92^{\circ}\right)$ is shown.

dependence on the altitude, $h$, above the photosphere. We adopted the formula

$\frac{\mathrm{d} N_{h}}{\mathrm{~d} h} \sim \exp \left(-\frac{h}{h_{0}}\right)$

where the scale-height $h_{0}=(11 \pm 3) \times 10^{3} \mathrm{~km}$ was pointed out by Catalano \& Van Allen (1973) for soft X-rays. The similar scaleheight was inferred from the investigation of the peak time differences between the soft and hard X-ray emission of 859 flares (Li \& Gan 2006). The obtained value $(7.7 \pm 1.3) \%$ agrees almost perfectly with the true population $(7.6 \%)$ in the catalogue. We conclude that our survey is almost complete.

\section{Results}

We chose to characterize each event by its appearance during a single time interval given in the HXT Flare Catalogue. This time interval was chosen to contain the strongest hard X-ray signal. Its duration depended on both the number of counts and the imaging requirements, and was thus, sometimes only a few seconds around a single burst, and other times, many minutes with many bursts (see Cols. (3) and (14) in Table 2).

\subsection{Power-law spectra}

Within this time interval, the total signal above the background within the entire image was accumulated in each energy band, which meant that our results were unaffected by the disadvantages of imaging spectroscopy. We calculated flux ratios between consecutive energy bands: M1/L, M2/M1, and H/M2, the so-called hardness ratios. From the hardness ratios, the average power-law indices in some energy ranges of the hard X-ray spectrum were obtained: $\mathrm{M} 1 / \mathrm{L} \rightarrow \gamma 1$, between 14 and $33 \mathrm{keV}$; $\mathrm{M} 2 / \mathrm{M} 1 \rightarrow \gamma 2$, between 23 and $53 \mathrm{keV}$; and H/M2 $\rightarrow \gamma 3$, between 33 and $93 \mathrm{keV}$.

Results are given in Cols. (11)-(13) of Table 2. Many events showed a signal above the background only for the low-energy bands, L and M1, thus for them there are no values of $\gamma 2$ and $\gamma 3$ in the table. For three flares, even the value of $\gamma 1$ was not 
Table 2. List of partially occulted flares found in the YOHKOH HXT Flare Catalogue ${ }^{a}$.

\begin{tabular}{|c|c|c|c|c|c|c|c|c|c|c|c|c|c|}
\hline 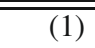 & (2) & (3) & (4) & (5) & (6) & (7) & (8) & (9) & (10) & (11) & (12) & (13) & (14) \\
\hline 00180 & $1991 / 10 / 21$ & $12: 51: 00+38$ & C7.8 & 6891 & S12E92.5 & 0.8 & 4.6 & 1.0 & 2.5 & 8.0 & $\ldots$ & $\ldots$ & $\mathrm{e}, \mathrm{o}, \mathrm{s}$ \\
\hline 01050 & $1991 / 11 / 30$ & $19: 07: 01+288$ & M1.0 & 6952 & N21E108.4 & 1.2 & 34.4 & 1.0 & 42.0 & 9.4 & $\ldots$ & $\ldots$ & $\mathrm{m}$ \\
\hline 01060 & $1991 / 12 / 02$ & $04: 53: 11+76$ & M3.6 & 6952 & N18E91.5 & 2.4 & 2.8 & 4.3 & 7.9 & 5.5 & 5.0 & $\ldots$ & $\mathrm{m}$ \\
\hline 01320 & 1991/12/09 & $02: 03: 08+57$ & M1.4 & 6966 & S06E91.0 & 1.0 & 1.9 & 2.0 & 3.7 & 9.5 & $\ldots$ & $\ldots$ & $\mathrm{e}, \mathrm{o}, \mathrm{s}$ \\
\hline 01340 & 1991/12/09 & $04: 19: 09+242.5$ & M3.6 & 6966 & S06E89.8 & 1.0 & 0 & 3.5 & 6.2 & 10.6 & $\ldots$ & $\ldots$ & $\mathrm{e}, \mathrm{m}, \mathrm{o}$ \\
\hline 02270 & $1992 / 01 / 13$ & $19: 04: 35+349.5$ & M1.3 & 7012 & S10E95.3 & 0.9 & 10.1 & 4.9 & 11.8 & 9.9 & $\ldots$ & $\ldots$ & $\mathrm{m}$ \\
\hline 02860 & $1992 / 02 / 06$ & $20: 51: 22+240.5$ & M4.1 & 7030 & N05W93.8 & 3.0 & 6.8 & 4.6 & 9.7 & 8.7 & $\ldots$ & $\ldots$ & $\mathrm{m}$ \\
\hline 03270 & $1992 / 02 / 19$ & $14: 45: 14+429$ & C9.4 & 7067 & N06E94.4 & 1.8 & 8.4 & 3.0 & 7.4 & 8.5 & $\ldots$ & $\ldots$ & $\mathrm{m}$ \\
\hline 04270 & $1992 / 06 / 28$ & $04: 47: 05+153.5$ & $\mathrm{X} 1.8$ & 7205 & N12W101.5 & 2.2 & 21.3 & 14.8 & 41.4 & 6.3 & 6.4 & $\ldots$ & $\mathrm{e}, \mathrm{s}$ \\
\hline 05090 & $1992 / 08 / 25$ & $19: 02: 52+160$ & $\mathrm{C} 8.7$ & 7260 & N13W98.2 & 2.0 & 15.2 & 14.1 & 32.7 & 8.5 & $\ldots$ & $\ldots$ & $\mathrm{s}$ \\
\hline 05210 & $1992 / 09 / 06$ & $09: 20: 11+27$ & C8.3 & 7276 & N15E101.4 & 0.5 & 21.1 & 1.0 & 16.3 & 6.1 & $\ldots$ & $\ldots$ & $\mathrm{s}$ \\
\hline 05260 & $1992 / 09 / 06$ & $23: 37: 17+29$ & M1.3 & 7276 & N17E93.6 & 2.6 & 6.4 & 1.0 & 3.2 & 8.3 & 7.5 & $\ldots$ & $\mathrm{o}, \mathrm{s}$ \\
\hline 05590 & $1992 / 10 / 05$ & $09: 24: 28+47.5$ & M2.0 & 7293 & S08W89.7 & 3.9 & 0 & 2.0 & 3.6 & 4.3 & 4.3 & $\ldots$ & $\mathrm{o}, \mathrm{s}$ \\
\hline 05920 & $1992 / 10 / 27$ & $22: 17: 44+104.5$ & C5.4 & 7315 & N07W88.5 & 1.5 & 0 & 4.1 & 7.5 & 7.6 & $\ldots$ & $\ldots$ & $\mathrm{m}, \mathrm{o}$ \\
\hline 06230 & 1992/11/01 & $11: 44: 06+53.5$ & $\mathrm{C} 4.9$ & 7321 & S25W89.3 & 2.2 & 0 & 11.9 & 21.2 & 5.9 & 8.7 & $\ldots$ & $\mathrm{o}, \mathrm{s}$ \\
\hline 06240 & $1992 / 11 / 02$ & $02: 59: 49+42$ & $X 9.0$ & 7321 & S25W97.9 & 2.3 & 14.2 & 14.6 & 33.6 & 8.0 & 7.3 & 6.0 & $\mathrm{e}, \mathrm{g}, \mathrm{s}$ \\
\hline 06280 & $1992 / 11 / 05$ & $20: 30: 17+167.5$ & $\mathrm{C} 8.2$ & 7323 & S17W91.5 & 2.0 & 2.6 & 5.0 & 9.1 & 8.7 & $\ldots$ & $\ldots$ & $\mathrm{s}$ \\
\hline 06440 & $1992 / 11 / 24$ & $10: 01: 02+157$ & C6.9 & 7342 & S07W95.5 & 5.0 & 7.4 & 3.0 & 8.6 & 6.5 & $\ldots$ & $\ldots$ & $\mathrm{s}$ \\
\hline 06480 & $1992 / 11 / 24$ & $20: 31: 03+271.5$ & C6.4 & 7342 & S07W101.2 & 5.2 & 17.9 & 1.5 & 16.3 & 8.7 & $\ldots$ & $\ldots$ & $\mathrm{e}, \mathrm{m}$ \\
\hline 06810 & $1993 / 02 / 01$ & $01: 57: 59+151$ & M2.2 & 7416 & S10E92.6 & 1.7 & 4.6 & 1.0 & 2.5 & 6.9 & 4.6 & $\ldots$ & $\mathrm{m}, \mathrm{o}$ \\
\hline 06820 & 1993/02/01 & $06: 58: 25+119.5$ & M1.4 & 7416 & S09E89.8 & 2.3 & 0.0 & 0.5 & 0.9 & 9.9 & $\ldots$ & $\ldots$ & $\mathrm{e}, \mathrm{m}, \mathrm{o}$ \\
\hline 07580 & $1993 / 03 / 15$ & $19: 07: 00+138.5$ & C5.5 & 7440 & S06W91.6 & 2.0 & 3.0 & 3.5 & 6.5 & 7.5 & $\ldots$ & $\ldots$ & $\mathrm{m}$ \\
\hline 07590 & $1993 / 03 / 15$ & $21: 05: 53+479$ & M3.0 & 7440 & S03W92.7 & 2.0 & 4.9 & 29.5 & 53.3 & 10.5 & $\ldots$ & $\ldots$ & $\mathrm{e}, \mathrm{m}$ \\
\hline 07680 & $1993 / 03 / 24$ & $03: 21: 45+42$ & C6.6 & 7448 & N15W92.1 & 3.7 & 3.8 & 2.7 & 5.3 & 6.2 & 7.4 & $\ldots$ & $\mathrm{s}$ \\
\hline 08220 & $1993 / 06 / 24$ & $14: 54: 48+176$ & C5.9 & 7530 & S11E90.9 & 0.7 & 1.7 & 1.0 & 1.9 & 9.8 & $\ldots$ & $\ldots$ & $\mathrm{m}, \mathrm{o}$ \\
\hline 08230 & $1993 / 06 / 24$ & $17: 27: 32+132.5$ & M4.2 & 7530 & S11E89.5 & 0.7 & 0 & 4.6 & 8.2 & 10.8 & $\ldots$ & $\ldots$ & $\mathrm{e}, \mathrm{s}$ \\
\hline 08460 & $1993 / 09 / 26$ & $10: 26: 04+63$ & $\mathrm{C} 3.4$ & 7590 & N14E98.0 & 1.1 & 14.0 & 1.0 & 8.8 & 7.1 & $\ldots$ & $\ldots$ & $\mathrm{e}, \mathrm{s}$ \\
\hline 08470 & $1993 / 09 / 26$ & $17: 26: 03+61$ & $\mathrm{C} 3.4$ & 7590 & N14E94.0 & 1.1 & 7.0 & 1.0 & 3.5 & 7.6 & $\ldots$ & $\ldots$ & $\mathrm{s}$ \\
\hline 08480 & $1993 / 09 / 26$ & $18: 29: 18+54.5$ & $\mathrm{C} 2.6$ & 7590 & N14E93.4 & 1.1 & 5.9 & 1.1 & 3.2 & 5.6 & 5.7 & $\ldots$ & $\mathrm{s}$ \\
\hline 09650 & $1994 / 01 / 29$ & $04: 10: 16+132$ & M2.4 & 7654 & N05W92.9 & 1.9 & 5.2 & 3.0 & 6.2 & $\ldots$ & $\ldots$ & $\ldots$ & $\mathrm{c}, \mathrm{s}$ \\
\hline 09660 & $1994 / 01 / 29$ & $11: 24: 49+80.5$ & M2.4 & 7654 & N07W97.0 & 1.9 & 12.4 & 6.5 & 16.9 & 7.0 & 8.1 & $\ldots$ & $\mathrm{m}$ \\
\hline 09700 & $1994 / 02 / 27$ & $09: 06: 47+541$ & M2.8 & 7671 & N08W97.5 & 3.8 & 13.6 & 12.6 & 38.6 & 7.9 & $\ldots$ & $\ldots$ & $\mathrm{h}, \mathrm{m}$ \\
\hline 11090 & $1997 / 11 / 25$ & $05: 34: 45+162.5$ & C5.0 & 8113 & N21E96.4 & 0.7 & 12.2 & 8.9 & 20.6 & 8.0 & $\ldots$ & $\ldots$ & $\mathrm{s}$ \\
\hline 11100 & $1997 / 11 / 25$ & $14: 40: 44+37.5$ & $\mathrm{C} 1.1$ & 8113 & N21E91.6 & 0.6 & 3.1 & 0.5 & 1.2 & 5.6 & $\ldots$ & $\ldots$ & $\mathrm{o}, \mathrm{s}$ \\
\hline 11290 & 1998/01/03 & $17: 13: 25+127$ & M2.7 & 8124 & S20W102.0 & 2.8 & 22.0 & 3.5 & 22.9 & 7.1 & 7.8 & $\ldots$ & $\mathrm{m}$ \\
\hline 11510 & $1998 / 03 / 23$ & $02: 59: 31+121.5$ & M2.3 & 8179 & S22W99.3 & 1.9 & 16.6 & 15.7 & 38.3 & 8.4 & $\ldots$ & $\ldots$ & $\mathrm{s}$ \\
\hline 11530 & $1998 / 03 / 24$ & $01: 47: 46+60$ & $\mathrm{C} 2.3$ & 8180 & S32W98.9 & 3.0 & 16.7 & 10.8 & 29.4 & 5.8 & $\ldots$ & $\ldots$ & $\mathrm{s}$ \\
\hline 11540 & $1998 / 03 / 24$ & $04: 42: 15+75$ & $\mathrm{C} 4.3$ & 8180 & S32W 100.5 & 3.0 & 19.7 & 10.0 & 32.0 & 6.3 & $\ldots$ & $\ldots$ & $\mathrm{s}$ \\
\hline 11650 & $1998 / 04 / 23$ & $05: 39: 55+151$ & $\mathrm{X} 1.2$ & 8210 & S18E103.7 & 0.7 & 25.5 & 2.2 & 30.0 & 7.7 & 7.7 & 2.4 & $\mathrm{~g}, \mathrm{~s}$ \\
\hline 11660 & $1998 / 04 / 24$ & $08: 46: 23+74.5$ & C8.9 & 8210 & S20E91.3 & 2.2 & 2.3 & 13.5 & 24.2 & 4.7 & 5.1 & $\ldots$ & $\mathrm{s}$ \\
\hline 11910 & $1998 / 05 / 08$ & $14: 20: 54+67.5$ & M1.8 & 8210 & S17W95.2 & 1.5 & 9.7 & 7.4 & 16.2 & 10.2 & $\ldots$ & $\ldots$ & $\mathrm{e}, \mathrm{s}$ \\
\hline 11930 & 1998/05/09 & $00: 17: 53+22.5$ & C8.3 & 8210 & S14W100.6 & 1.6 & 19.7 & 1.1 & 14.4 & 6.3 & $\ldots$ & $\ldots$ & $\mathrm{s}$ \\
\hline 11950 & $1998 / 05 / 09$ & $03: 22: 56+70.5$ & M7.7 & 8210 & S17W102.3 & 1.6 & 22.8 & 1.4 & 19.6 & 6.8 & 6.1 & 3.9 & $\mathrm{~g}, \mathrm{~h}, \mathrm{~s}$ \\
\hline 12000 & $1998 / 05 / 10$ & $08: 26: 33+113$ & M1.6 & 8220 & S27E91.4 & 2.4 & 2.6 & 4.5 & 8.2 & 9.8 & $\ldots$ & $\ldots$ & $\mathrm{e}, \mathrm{s}$ \\
\hline 12010 & $1998 / 05 / 10$ & $13: 18: 30+14$ & M3.9 & 8220 & S27E88.8 & 2.4 & 0 & 1.1 & 2.1 & 4.9 & 3.5 & 3.3 & $\mathrm{e}, \mathrm{o}, \mathrm{s}$ \\
\hline 13580 & $1998 / 11 / 22$ & $12: 21: 38+317.5$ & $\mathrm{C} 8.8$ & 8393 & S17E93.4 & 4.2 & 6.2 & 3.0 & 6.6 & 8.8 & $\ldots$ & $\ldots$ & $\mathrm{m}$ \\
\hline 13610 & $1998 / 11 / 23$ & $05: 58: 41+39.5$ & $\mathrm{C} 4.9$ & 8384 & S29W92.3 & 2.0 & 4.3 & 4.2 & 8.1 & 6.1 & 7.5 & $\ldots$ & $\mathrm{s}$ \\
\hline 13620 & $1998 / 11 / 23$ & $06: 53: 06+5.5$ & $\mathrm{X} 2.2$ & 8384 & S29W92.8 & 2.0 & 5.2 & 8.3 & 15.7 & 10.3 & $\ldots$ & $\ldots$ & $\mathrm{e}, \mathrm{s}$ \\
\hline 13650 & $1998 / 11 / 24$ & $02: 17: 48+316.5$ & $\mathrm{X} 1.0$ & 8384 & S30W103.1 & 2.0 & 24.5 & 3.2 & 27.3 & 8.8 & 9.6 & 4.5 & $\mathrm{e}, \mathrm{g}, \mathrm{m}$ \\
\hline 15640 & $1999 / 06 / 17$ & $17: 20: 47+56.5$ & M3.6 & 8584 & N22W?? & $\ldots$ & $>0$ & 5.9 & $>10.5$ & 7.7 & $\ldots$ & $\ldots$ & $\mathrm{e}, \mathrm{s}$ \\
\hline 16290 & $1999 / 07 / 23$ & $05: 01: 27+44$ & C9.4 & 8645 & S23E97.4 & 3.3 & 13.8 & 4.0 & 13.5 & 6.8 & $\ldots$ & $\ldots$ & $\mathrm{s}$ \\
\hline 16310 & $1999 / 07 / 23$ & $15: 56: 13+21.5$ & M1.0 & 8645 & S26E91.6 & 3.3 & 3.0 & 1.1 & 2.3 & 5.5 & 6.5 & $\ldots$ & $\mathrm{o}, \mathrm{s}$ \\
\hline 16810 & $1999 / 08 / 07$ & $19: 13: 10+309$ & M1.2 & 8645 & S20W103.1 & 3.3 & 24.4 & 9.3 & 36.8 & 10.9 & $\ldots$ & $\ldots$ & $\mathrm{e}, \mathrm{s}$ \\
\hline 16820 & $1999 / 08 / 07$ & $20: 54: 08+741$ & M1.7 & 8645 & S29W104.0 & 3.3 & 26.1 & 3.9 & 31.5 & 10.0 & $\cdots$ & $\ldots$ & $\mathrm{m}$ \\
\hline 17400 & $1999 / 10 / 01$ & $00: 15: 28+60.5$ & C7.7 & 8716 & N22E95.8 & 2.2 & 10.7 & 1.1 & 5.8 & 7.8 & $\ldots$ & $\ldots$ & s \\
\hline 17710 & $1999 / 10 / 27$ & 09:09:39+90.5 & M1.0 & 8737 & S12W87.8 & 5.9 & 0 & 1.8 & 3.2 & 6.0 & 4.4 & $\ldots$ & $\mathrm{o}, \mathrm{s}$ \\
\hline 17720 & $1999 / 10 / 27$ & $13: 28: 21+535$ & M1.8 & 8737 & S15W90.3 & 6.0 & 0.6 & 4.0 & 7.1 & 9.1 & $\ldots$ & $\ldots$ & $\mathrm{m}$ \\
\hline
\end{tabular}


Table 2. continued.

\begin{tabular}{|c|c|c|c|c|c|c|c|c|c|c|c|c|c|}
\hline (1) & (2) & (3) & (4) & (5) & (6) & (7) & (8) & (9) & (10) & (11) & (12) & (13) & (14) \\
\hline 17730 & 1999/10/27 & $15: 28: 00+762$ & M1.4 & 8737 & S14W91.6 & 6.0 & 2.6 & 11.5 & 20.7 & 9.9 & $\cdots$ & $\ldots$ & $\mathrm{m}$ \\
\hline 17790 & 1999/11/05 & $18: 16: 47+34$ & M3.0 & 8759 & N12E95.8 & 1.3 & 11.0 & 7.0 & 16.2 & 8.4 & $\ldots$ & $\ldots$ & $\mathrm{e}, \mathrm{s}$ \\
\hline 18610 & 1999/12/17 & $00: 19: 22+74$ & C7.0 & 8806 & N19E91.8 & 0.7 & 3.4 & 1.0 & 2.1 & 7.9 & 7.8 & $\ldots$ & $\mathrm{o}, \mathrm{s}$ \\
\hline 19030 & 2000/01/18 & 09:37:03+299 & M1.2 & 8827 & S15W105.6 & 3.1 & 28.2 & 1.8 & 30.9 & 6.8 & 7.0 & $\ldots$ & $\mathrm{m}$ \\
\hline 19630 & 2000/03/07 & $03: 48: 58+24$ & $\mathrm{C} 2.9$ & 8906 & S16E95.9 & 6.7 & 10.7 & 1.6 & 6.7 & 7.0 & $\ldots$ & $\ldots$ & $\mathrm{o}, \mathrm{s}$ \\
\hline 20920 & $2000 / 05 / 03$ & $22: 58: 52+741$ & M1.1 & 8970 & S18W90.1 & 2.2 & 0.0 & 7.4 & 13.2 & 9.3 & $\ldots$ & $\ldots$ & $\mathrm{m}$ \\
\hline 20930 & $2000 / 05 / 05$ & $15: 18: 42+1954$ & M1.5 & 8970 & S18W110.1 & 3.7 & 37.5 & 2.2 & 51.6 & 8.5 & 4.7 & $\ldots$ & $\mathrm{m}$ \\
\hline 20980 & $2000 / 05 / 12$ & $08: 41: 48+85$ & C8.1 & 8998 & S14E90.5 & 3.9 & 0.9 & 3.6 & 6.4 & 9.1 & $\ldots$ & $\ldots$ & $\mathrm{s}$ \\
\hline 21030 & $2000 / 05 / 13$ & $01: 36: 19+232$ & M1.1 & 9002 & N22E108.8 & 0.8 & 33.8 & 4.9 & 51.5 & 9.5 & $\ldots$ & $\ldots$ & $\mathrm{e}, \mathrm{s}$ \\
\hline 21050 & 2000/05/14 & $00: 25: 08+97.5$ & C7.5 & 9002 & N22E96.1 & 0.7 & 11.0 & 4.5 & 12.3 & 7.2 & . & $\ldots$ & $\mathrm{e}, \mathrm{s}$ \\
\hline 21280 & 2000/05/17 & $04: 02: 46+14$ & C7.1 & 8993 & S20W90.4 & 1.7 & 0.7 & 2.0 & 3.6 & 4.6 & 5.0 & $\ldots$ & $\mathrm{o}, \mathrm{s}$ \\
\hline 21530 & $2000 / 05 / 24$ & $03: 13: 26+83.5$ & C7.0 & 9017 & S12E92.9 & 3.0 & 5.3 & 2.1 & 4.6 & 7.9 & . & $\ldots$ & $\mathrm{o}, \mathrm{s}$ \\
\hline 21880 & 2000/06/12 & $01: 37: 32+10$ & C6.1 & 9042 & N21E91.2 & 7.8 & 2.2 & 1.6 & 3.0 & 5.8 & 4.1 & $\ldots$ & $\mathrm{o}, \mathrm{s}$ \\
\hline 22570 & $2000 / 07 / 15$ & $04: 33: 29+32$ & $\mathrm{C} 2.7$ & 9090 & N14E94.8 & 0.8 & 8.5 & 0.5 & 3.4 & & & $\ldots$ & $1, \mathrm{~s}$ \\
\hline 23070 & $2000 / 07 / 27$ & $16: 46: 58+111$ & M1.5 & 9087 & S09W105.4 & 1.2 & 26.9 & 5.7 & 36.7 & 7.2 & 8.4 & $\ldots$ & $\mathrm{s}$ \\
\hline 23590 & 2000/09/22 & $23: 46: 52+69$ & C8.5 & 9165 & N14W94.5 & 1.5 & 8.0 & 4.6 & 10.4 & 5.6 & 6.6 & $\ldots$ & $\mathrm{s}$ \\
\hline 23750 & $2000 / 09 / 30$ & $20: 15: 37+17.5$ & M1.8 & 9169 & N07W90.8 & 2.3 & 1.4 & 3.6 & 6.5 & 9.2 & $\ldots$ & $\ldots$ & $\mathrm{e}, \mathrm{s}$ \\
\hline 23780 & 2000/10/01 & $07: 01: 39+26$ & M5.0 & 9169 & N08W96.8 & 2.4 & 12.2 & 10.6 & 23.9 & 10.2 & $\ldots$ & $\ldots$ & $\mathrm{e}, \mathrm{s}$ \\
\hline 23790 & 2000/10/01 & 14:00:08+56 & M2.2 & 9169 & N09W100.7 & 2.4 & 19.2 & 2.1 & 16.3 & 6.0 & 6.6 & $\ldots$ & $\mathrm{m}$ \\
\hline 23810 & 2000/10/02 & $02: 17: 44+23$ & C5.0 & 9182 & N01E89.6 & 1.4 & 0 & 2.0 & 3.6 & 4.8 & 4.6 & $\ldots$ & $\mathrm{o}, \mathrm{s}$ \\
\hline 23940 & 2000/10/16 & $05: 41: 56+166$ & C7.0 & 9182 & N04W107.4 & 3.3 & 30.1 & 3.0 & 39.0 & 7.1 & 9.6 & $\ldots$ & $\mathrm{m}$ \\
\hline 23950 & 2000/10/16 & $06: 59: 48+1018.5$ & M2.5 & 9182 & N03W108.3 & 3.3 & 31.5 & 4.0 & 44.6 & 10.8 & $\ldots$ & $\ldots$ & s \\
\hline 24480 & 2000/12/08 & $16: 18: 57+90.5$ & C4.3 & 9246 & S09W90.6 & 1.3 & 1.1 & 2.1 & 3.8 & 7.0 & ... & $\ldots$ & $\mathrm{m}, \mathrm{o}$ \\
\hline 24580 & 2000/12/18 & $20: 12: 57+27$ & $\mathrm{C} 2.7$ & 9280 & N08E92.5 & 0.5 & 4.4 & 2.0 & 4.2 & 9.3 & $\ldots$ & $\ldots$ & $\mathrm{o}, \mathrm{s}$ \\
\hline 24840 & 2001/01/03 & $18: 05: 31+44.5$ & $\mathrm{C} 2.7$ & 9302 & N20E97.2 & 1.2 & 12.8 & 9.0 & 22.0 & $\cdots$ & $\cdots$ & $\ldots$ & $\mathrm{c}, \mathrm{s}$ \\
\hline 25540 & 2001/04/01 & $11: 42: 36+36$ & M5.5 & 9415 & S21E107.0 & 0.7 & 30.3 & 23.6 & 77.9 & 9.2 & 6.7 & $\ldots$ & $\mathrm{e}, \mathrm{h}, \mathrm{s}$ \\
\hline 25870 & $2001 / 04 / 05$ & $02: 06: 55+75$ & M3.1 & 9393 & N15W99.2 & 1.6 & 16.3 & 3.2 & 15.2 & 6.1 & 6.8 & $\ldots$ & $\mathrm{s}$ \\
\hline 25880 & 2001/04/05 & $05: 12: 45+119$ & M1.1 & 9393 & N15W100.8 & 1.6 & 19.3 & 4.6 & 21.3 & 8.1 & 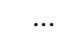 & $\ldots$ & $\mathrm{m}$ \\
\hline 25890 & 2001/04/05 & 08:33:08+8 & M8.4 & 9393 & N14W102.9 & 1.7 & 23.1 & 6.3 & 30.1 & 8.3 & 6.6 & $\ldots$ & $\mathrm{e}, \mathrm{s}$ \\
\hline 26060 & 2001/04/18 & $02: 14: 29+36.5$ & $\mathrm{C} 2.2$ & 9415 & S21W116.9 & 1.8 & 47.9 & 9.0 & 108.4 & 3.4 & 3.7 & 3.7 & $\mathrm{~g}, \mathrm{~s}$ \\
\hline 26480 & $2001 / 05 / 20$ & $06: 02: 17+6.5$ & M6.4 & 9455 & S17W94.4 & 2.6 & 8.1 & 0.7 & 3.4 & 4.0 & 3.4 & 3.6 & $\mathrm{o}, \mathrm{s}$ \\
\hline 26490 & $2001 / 05 / 20$ & $09: 19: 43+51.5$ & M1.5 & 9455 & S20W96.2 & 2.6 & 11.3 & 1.0 & 6.1 & 8.5 & 5.8 & $\ldots$ & $\mathrm{m}$ \\
\hline 27080 & 2001/08/09 & $00: 39: 15+120$ & C6.0 & 9557 & S19W91.7 & 3.3 & 3.1 & 5.4 & 9.9 & 6.6 & 7.9 & $\ldots$ & $\mathrm{s}$ \\
\hline 27400 & $2001 / 08 / 29$ & $09: 12: 02+59.5$ & C6.5 & 9587 & S12W97.4 & 3.5 & 12.7 & 1.0 & 7.8 & 6.7 & 8.3 & $\ldots$ & $\mathrm{s}$ \\
\hline 27500 & 2001/09/03 & $01: 54: 38+133$ & C9.0 & 9607 & S15E93.1 & 3.3 & 5.6 & 3.4 & 7.1 & 10.1 & $\ldots$ & $\ldots$ & $\mathrm{s}$ \\
\hline 27540 & 2001/09/03 & $18: 23: 03+193.5$ & M2.5 & 9608 & S22E96.2 & 1.0 & 12.2 & 1.4 & 6.9 & 8.1 & 6.9 & $\ldots$ & $\mathrm{m}$ \\
\hline 27890 & 2001/09/11 & 07:00:00+69 & M1.2 & 9616 & S10E92.3 & 4.4 & 4.1 & 6.8 & 12.7 & 8.0 & 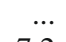 & $\ldots$ & $\mathrm{s}$ \\
\hline 28260 & 2001/09/19 & $06: 54: 11+56.5$ & C7.2 & 9608 & S23W93.9 & 2.2 & 7.6 & 10.2 & 19.9 & 5.6 & 7.2 & ... & $\mathrm{s}$ \\
\hline 29110 & $2001 / 10 / 29$ & $01: 56: 00+41.5$ & M1.3 & 9669 & N12W103.9 & 3.0 & 25.5 & 2.5 & 26.0 & 5.3 & 5.0 & $\ldots$ & $\mathrm{m}$ \\
\hline 30430 & 2001/12/01 & $15: 12: 21+847$ & M4.8 & 9727 & S25E97.8 & 2.4 & 14.3 & 13.6 & 31.6 & 9.0 & $\ldots$ & $\ldots$ & $\mathrm{e}, \mathrm{m}$ \\
\hline 30470 & 2001/12/02 & $21: 29: 50+251.5$ & M2.0 & 9714 & S11W108.1 & 2.9 & 32.5 & 8.6 & 53.0 & 9.7 & $\ldots$ & $\ldots$ & $\mathrm{m}$ \\
\hline
\end{tabular}

a Descriptions: (1) - catalogue event number; (2) - date (YYYY/MM/DD); (3) - time integration (start time [UT] + duration in seconds); (4) GOES X-ray class; (5) - NOAA active region number; (6) - location in solar coordinates; (7) - uncertainty of longitude estimation; (8) - estimated number of hours before/after the limb passage; (9) - altitude of HXR centroid above the solar limb in SXT pixels [2.45 arcsec]; (10) - estimated total height in $10^{3} \mathrm{~km} ;(11)-\gamma 1$, the power-law index from hardness ratio M1/L; (12) - $\gamma 2$, the power-law index from hardness ratio M2/M1; (13) $-\gamma 3$, the power-law index from hardness ratio H/M2; (14) - remarks: $\mathrm{c}$ - corrupted HXT file (no HXT diagnostics), e - maximum of flare outside the time integration, $\mathrm{g}$ - gamma rays, $\mathrm{h}$ - progressive spectral hardening, 1 - only channel L (no HXT diagnostics), $\mathrm{m}$ - multiple bursts, $\mathrm{o}-$ event situated too close to the solar limb, possibly footpoint contamination, $\mathrm{s}-$ single burst.

obtained: for two of them (Nos. 09650 and 24 840) source data files were corrupted, and for event No. 22570 the hard X-ray flux was recorded only in the channel L. Uncertainties in the $\gamma$ indices, estimated according to the number of counts using the Poisson statistics, were typically 0.1-0.2.

In Fig. 3, two kinds of flares not included in our statistical analyses are marked. The events marked with boxes were not observed in the maximum of hard X-rays, and therefore values of $\gamma$ indices obtained for them are unrepresentative of the highest energy phase available for other events. For uniformity of results, these flares are omitted in the later analysis.

The events marked in Fig. 3 with stars probably occurred too close to the solar limb and the possibility of hard X-ray emission from their footpoints is not excluded completely. For these events a small hard X-ray source is indeed seen centered close to the solar limb (e.g., Fig. 1c). Its size is much smaller than a size of a typical coronal source. We found that these sources are seen basically for the longitudes below $92^{\circ}$. Our aim is to study the characteristics of coronal hard X-ray sources, therefore these flares are also omitted in our later analysis.

A histogram of the $\gamma 1$ index values obtained for 94 events in our survey is presented in Fig. 4, where also superimpose two separate histograms, one for events without the hard X-ray maximum (boxes in Fig. 3) and another for events with possible footpoint contamination (stars in Fig. 3). With respect to our expectations, the first histogram, which contains the less energetic events, is shifted towards higher values, while the second, including more energetic events, is shifted towards lower values. We conclude that because of strict selection criteria applied, the final population is more uniform. 


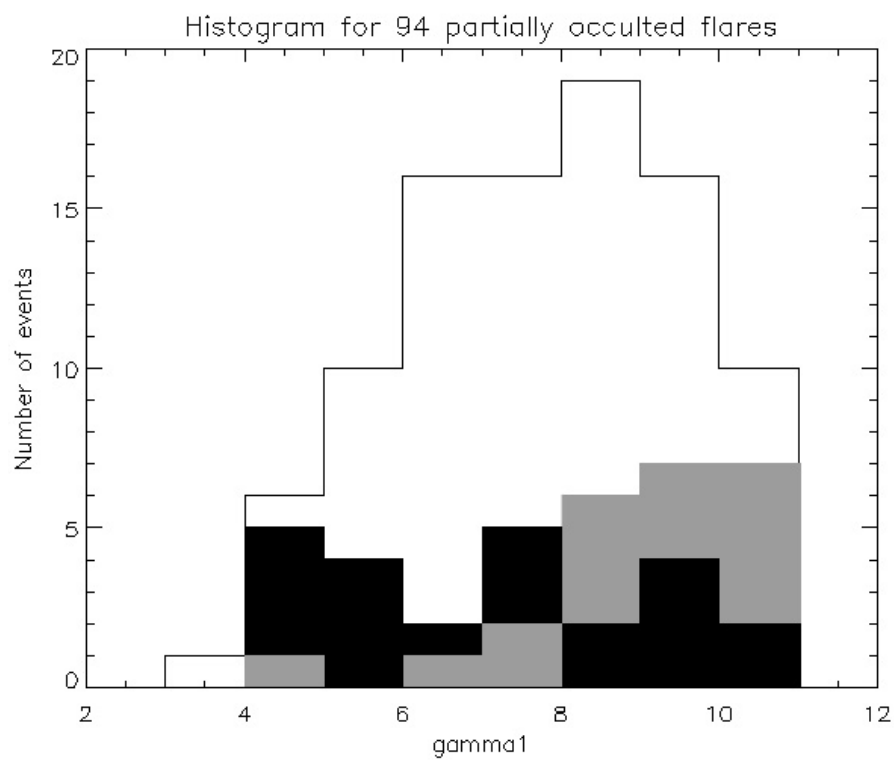

Fig. 4. Histogram of values of power-law index obtained for 94 partially occulted flares in energy range $14-33 \mathrm{keV}$. Gray bins represent 24 events that were not observed in the maximum of hard X-rays, black bins represent 24 events with possibly footpoint contamination.

We present the final histogram in Fig. 5a. It contains values for 52 partially occulted flares remaining after rejection of the two groups of events described in the previous paragraph. The histogram of values of the $\gamma 1$ index obtained for 620 nonocculted flares identified in the catalogue is given in the same panel. For straightforward comparison, both histograms are normalized. As we can see, the central parts of the histograms, containing about two third of events, are shifted with respect to each other. The average value for partially occulted flares is 7.5, while the average value for non-occulted flares is 4.8. Standard deviations for both histograms are 1.6.

In Fig. 5b, we compare normalized histograms of the $\gamma 2$ index obtained for partially occulted and non-occulted flares, respectively. Because part of the flare signal above the background in energy band M2 was imperceptible, the total number of events decreased to 23 and 338, respectively. The central parts of histograms, which contain more than $70 \%$ of the events, are shifted one from another. The average value for partially occulted flares is 6.7, and the average value for non-occulted flares is 4.0, while the standard deviations are 1.4 and 1.1 , respectively.

The calculation of the $\gamma 3$ index requires the detection of a distinctive signal above the background in energy band $\mathrm{H}$. This once more limits the number of events to 155 non-occulted and only 3 partially occulted flares. In Fig. $5 c$ we therefore present only the normalized histogram for non-occulted flares. The average value is 3.4 and the standard deviation is 0.7 . Three partially occulted flares emitting signal strong enough in energy band $\mathrm{H}$ show values of the $\gamma 3$ index similar to the typical values obtained for disk flares. They are: event No.11650 of 1998 April 23 (Sato 2001; Tomczak 2004), event No. 11950 of 1998 May 9 (Tomczak 2008), and event No. 26060 of 2001 April 18 (Hudson et al. 2001). We emphasize that all these events show very spectacular X-ray plasma ejections.

\subsection{Deviations from power-law spectra}

The histograms of power-law index values in Fig. 5, show a shift towards lower values with increasing photon energies for which
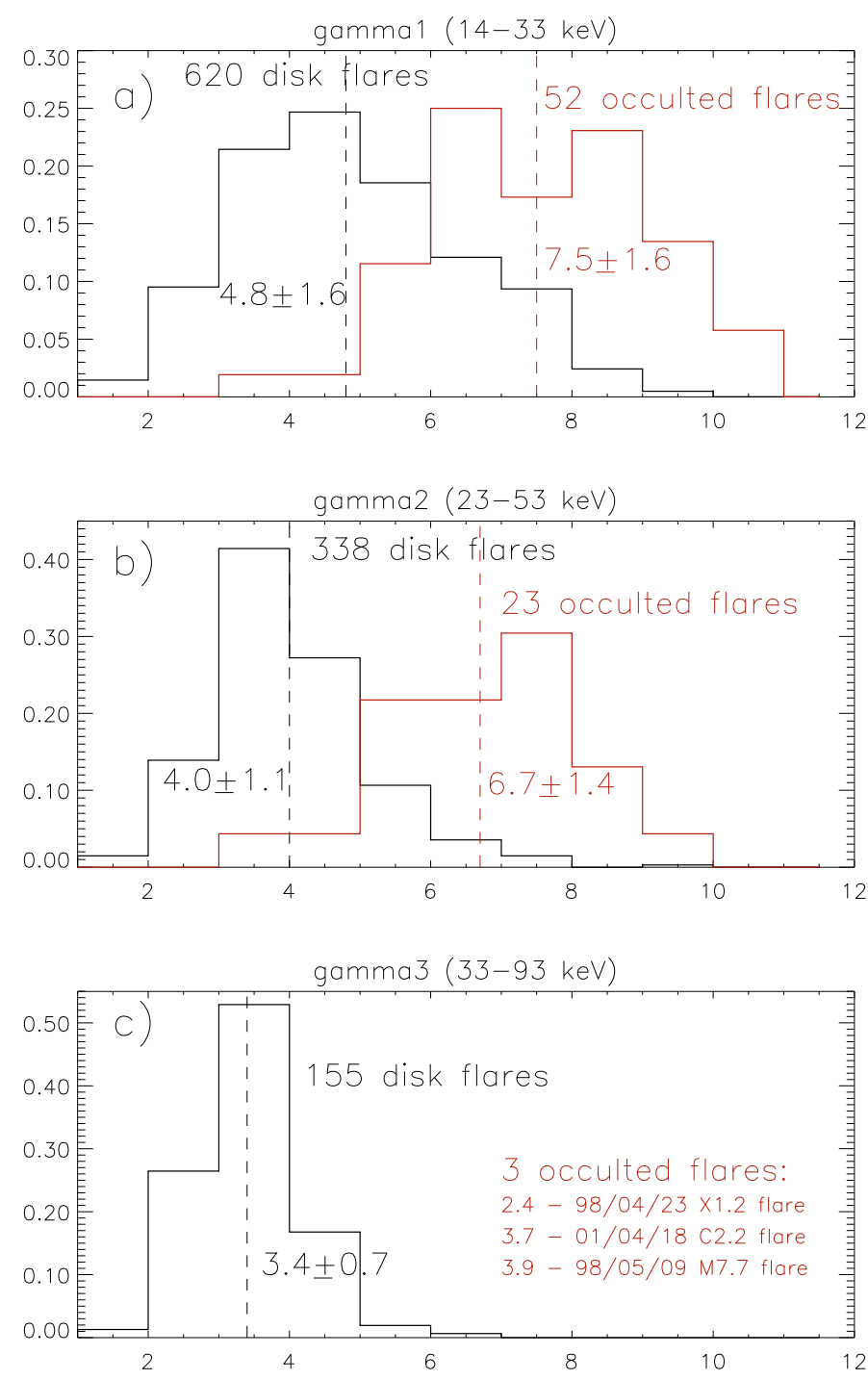

Fig. 5. Normalized histograms of values of the power-law index fitted in different ranges of the hard X-ray energy photon spectrum. Separate histograms for partially occulted and disk flares are presented. Energy ranges, number of events, average values and standard deviations are written.

the power-law was fitted. This shift is seen for partially occulted as well as non-occulted flares. This appearance is strongly indicative of a contamination of hard X-rays by the emission of thermal plasma, which is expected to decrease with photon energy.

The HXT provides poor spectral resolution (only four broad energy bands), which excludes the reasonable fitting of both a non-thermal power-law component and a thermal one. Therefore, another method for distinguishing between nonthermal and thermal components should be proposed. In this paper, we calculated the average power-law index $\gamma 1, \gamma 2$, and $\gamma 3$ for the energy ranges $14-33,23-53$, and 33-93 keV, respectively. If an investigated photon energy spectrum could be described by a single power-law formula, then the obtained values of $\gamma 1, \gamma 2$, and $\gamma 3$ should be similar within observational uncertainties. Some larger differences between these values strongly suggest a deviation from the single power-law caused, for example, by the presence of the thermal component.

In Fig. 6, we present the comparison of $\gamma 1$ and $\gamma 2$ for flares presented in the histograms of Fig. 5a,b. For events within the 


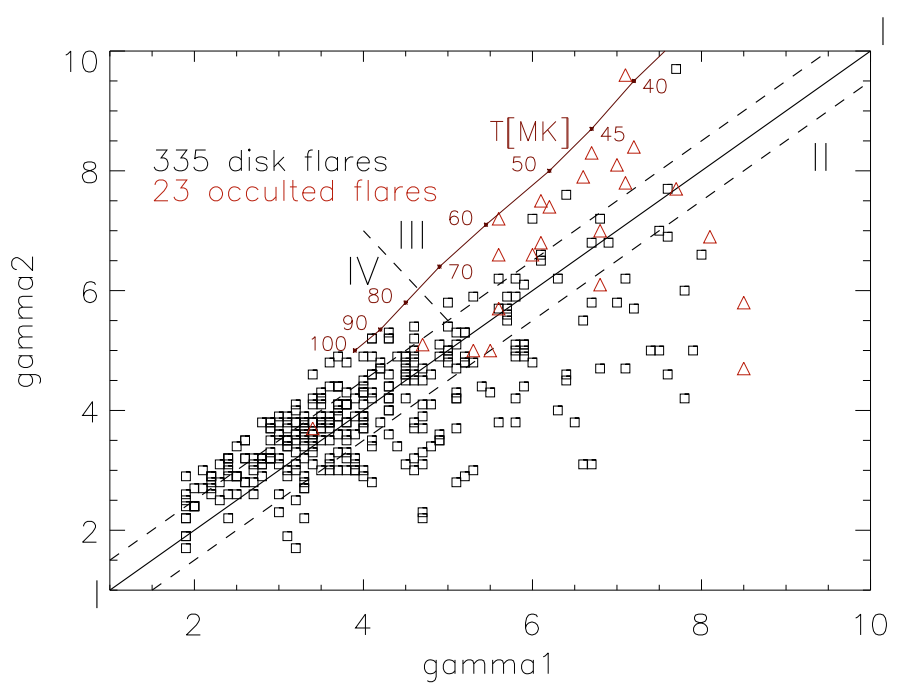

Fig. 6. Comparison between values of the power-law index fitted in two energy ranges: $14-33 \mathrm{keV}(\gamma 1)$ and $23-53 \mathrm{keV}(\gamma 2)$. Partially occulted flares are marked with triangles, non-occulted flares are marked with boxes. In dependence on mechanisms responsible for a hard X-ray spectrum formation the points are situated in one of four regions described by digits between I and IV. For additional explanations - see text. Statistics of occurrence is presented in Table 3. The solid narrow line refers to emission of purely thermal plasma with temperatures between 38 and $100 \mathrm{MK}$.

Table 3. Statistics of different types of spectra in the $14-53 \mathrm{keV}$ energy range.

\begin{tabular}{cccc}
\hline \hline $\begin{array}{c}\text { Area } \\
\text { in Fig. } 6\end{array}$ & $\begin{array}{c}\text { Type of } \\
\text { spectrum }\end{array}$ & $\begin{array}{c}\text { Partially } \\
\text { occulted }\end{array}$ & Non-occulted \\
\hline I & non-thermal & $30.4 \%$ & $55.2 \%$ \\
& $(\gamma 1 \sim \gamma 2)$ & $(7 / 23)$ & $(185 / 335)$ \\
II & thermal + non-thermal & $17.4 \%$ & $24.2 \%$ \\
& $(\gamma 1>\gamma 2)$ & $(4 / 23)$ & $(81 / 335)$ \\
III & quasi-thermal & $52.2 \%$ & $1.8 \%$ \\
& $(\gamma 1<\gamma 2)$ & $(12 / 23)$ & $(6 / 335)$ \\
IV & albedo & $0 \%$ & $18.8 \%$ \\
& $(\gamma 1<\gamma 2)$ & $(0 / 23)$ & $(63 / 335)$ \\
\hline
\end{tabular}

central region between the two straight dashed lines (area I), differences between $\gamma 1$ and $\gamma 2$ are below the expected uncertainties, i.e., $|\gamma 1-\gamma 2| \leq 0.5$. It means that in the energy range $14-53 \mathrm{keV}$, their spectra can be described by the single powerlaw. We consider them as purely non-thermal.

Area II below the central region is occupied by flares for which $(\gamma 1-\gamma 2)>0.5$. It means that their spectra are steeper at lower and flatter at higher energies. We explain this shape as the consequence of the thermal component presence in energy band $\mathrm{L}$.

The area above the central region is occupied by flares for which $(\gamma 2-\gamma 1)>0.5$, which means that their spectra are flatter at lower and steeper at higher energies. This shape suggests a thermal nature. However, purely thermal events should be located in the figure along the solid narrow line above the central region. As we can see, few events are close to this line. The majority of events located in area III is quite quasi-thermal, i.e., the thermal component dominates but is mixed with non-thermal bursts as reported by Tomczak (2001).

Events in area IV are too energetic to explain their spectra by means of thermal emission. Zhang \& Huang (2003) proposed that values of $\gamma 1$ lower than $\gamma 2$ can be caused by Compton

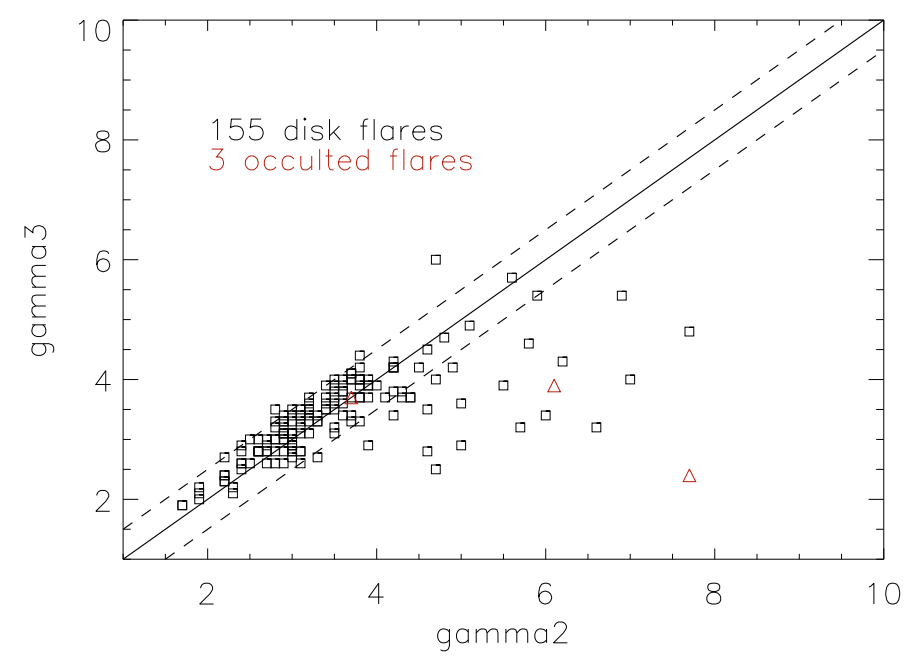

Fig. 7. Comparison between values of the power-law index fitted in two energy ranges: $23-53 \mathrm{keV}(\gamma 2)$ and 33-93 keV $(\gamma 3)$. Partially occulted flares are marked with triangles, non occulted flares are marked with boxes. Statistics of occurrence is presented in Table 4. For other explanations - see text.

backscattering. Known as the photospheric albedo, this effect produces a shift in photon energy from band L to higher energies. Kašparova et al. (2007) demonstrated that the photospheric albedo depends on the heliocentric distance. We checked and found that the longitudes of 38 events out of 50 in area IV, for which $\mathrm{H} \alpha$ coordinates are given in the catalogue, are below $45^{\circ}$. We consider this as being consistent with events situated in area IV having non-thermal spectra modified by the photospheric albedo.

Statistics of the occurrence of flares in different areas of Fig. 6 for partially occulted and non-occulted flares separately is given in Table 3. The results can be summarized as follows. During the hard X-ray maximum in energy band $\mathrm{L}$ (14-23 keV), the non-thermal component dominates about $74 \%$ of non-occulted flares and only about $30 \%$ of partially occulted flares. On the other hand, the thermal component dominates over $50 \%$ of partially occulted flares and only about $2 \%$ of nonocculted flares.

In Fig. 7, we present the comparison between values of $\gamma 2$ and $\gamma 3$ for flares from histograms in Fig. 5b,c. The shift in the investigation towards higher energies reduces the heterogeneity of the hard X-ray spectra evident in Fig. 6. The points are concentrated in areas I and II, which means that in the energy range $23-93 \mathrm{keV}$ only pure non-thermal spectra or thermal and non-thermal mixtures frequently occur. The statistical analysis is presented in Table 4. Only total results are given because there are few partially occulted events that show values similar to nonocculted flares. During the hard X-ray maximum in energy band M1 (23-33 keV), about $85 \%$ of flares show purely non-thermal spectra, but the thermal component remains in about $15 \%$ of events.

We check how deviations from power-law spectra have affected the values of power-law indices. The histograms of the power-law index for flares in area I of Figs. 6 and 7 are presented in Fig. 8. We expect these events to be free of disturbances introduced by the thermal component and the photospheric albedo. The histogram of 185 non-occulted events from Fig. 6 resembles the histogram shown in Fig. 5b. Their medians are $3.7 \pm 1.1$ and $4.0 \pm 1.1$, respectively. This small difference is caused by 
Table 4. Statistics of different types of spectra in the $23-93 \mathrm{keV}$ energy range

\begin{tabular}{ccccc}
\hline \hline $\begin{array}{c}\text { Area } \\
\text { in Fig. 7 }\end{array}$ & $\begin{array}{c}\text { Type of } \\
\text { spectrum }\end{array}$ & $\begin{array}{c}\text { Partially } \\
\text { occulted }\end{array}$ & $\begin{array}{c}\text { Non- } \\
\text { occulted }\end{array}$ \\
\hline I & $\begin{array}{c}\text { non-thermal } \\
(\gamma 2 \sim \gamma 3)\end{array}$ & $(1 / 3)$ & $82.9 \%$ & $(130 / 155)$ \\
II & $\begin{array}{c}\text { thermal }+ \text { non- } \\
\text { thermal }(\gamma 2>\gamma 3) \\
\text { quasi-thermal } \\
(\gamma 2<\gamma 3) \\
\text { III }\end{array}$ & $(2 / 3)$ & $15.2 \%$ & $(22 / 155)$ \\
IV & $\begin{array}{c}\text { albedo } \\
(\gamma 2<\gamma 3)\end{array}$ & $(0 / 3)$ & $0 \%$ & $(0 / 155)$ \\
& & & $1.9 \%$ & $(3 / 155)$ \\
\hline
\end{tabular}

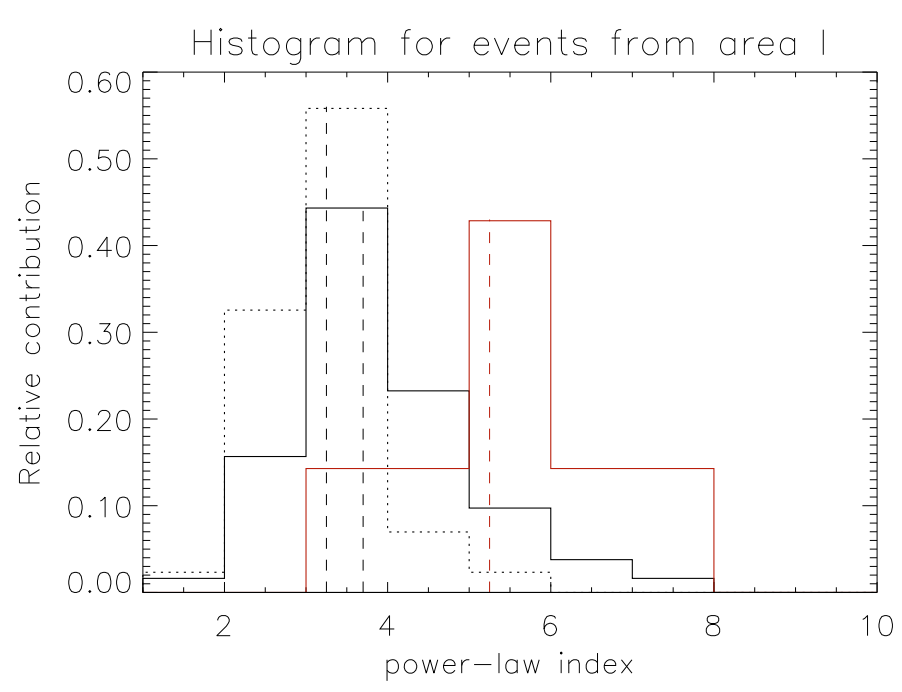

Fig. 8. Normalized histograms of values of the power-law index for flares situated in area I of Figs. 6 and 7 which are believed to be free of disturbances introduced by the thermal component and the photospheric albedo. Solid lines represent the data for energy range $14-53 \mathrm{keV}$ for partially occulted (red) and non-occulted (black) flares separately. The dotted line represents the data for energy range $23-93 \mathrm{keV}$ for nonocculted flares. Medians of histograms are marked with dashed lines.

the small amounts of thermally dominated flares in non-occulted events (see Table 3 ).

Partially occulted flares represents another case. Because of the frequent occurrence of thermally dominated events (Table 3), the histogram shown in Fig. 8 is clearly shifted towards lower values than the histogram presented in Fig. 5b. The medians are $5.2 \pm 1.4$ and $6.7 \pm 1.4$, respectively. In conclusion, after removing the contamination of hard X-rays with emission of thermal plasma, partially occulted flares in our survey exhibit higher values of the power-law index than non-occulted flares. However, the difference between the medians of histograms in Figs. 5b and 8 has decreased from 2.7 to 1.5 .

In this way, is it possible to eliminate the contribution of thermal emission completely? The comparison between histograms for non-occulted flares in Fig. 8 for two energy ranges of investigated spectra suggests that the interpretation should be completed with caution. The histogram obtained for higher energies is shifted slightly towards lower values of the power-law index. Its median is $3.2 \pm 0.7$, i.e., 0.5 lower than the median of the second histogram. This can be interpreted as the cause of a thermal contribution. However, one cannot exclude there being a broken power law with a break energy around $50 \mathrm{keV}$ (i.e., between energy bands $\mathrm{M} 2$ and $\mathrm{H}$ ) and a flatter slope above this value. This situation of an upward "knee" due to the transition from a

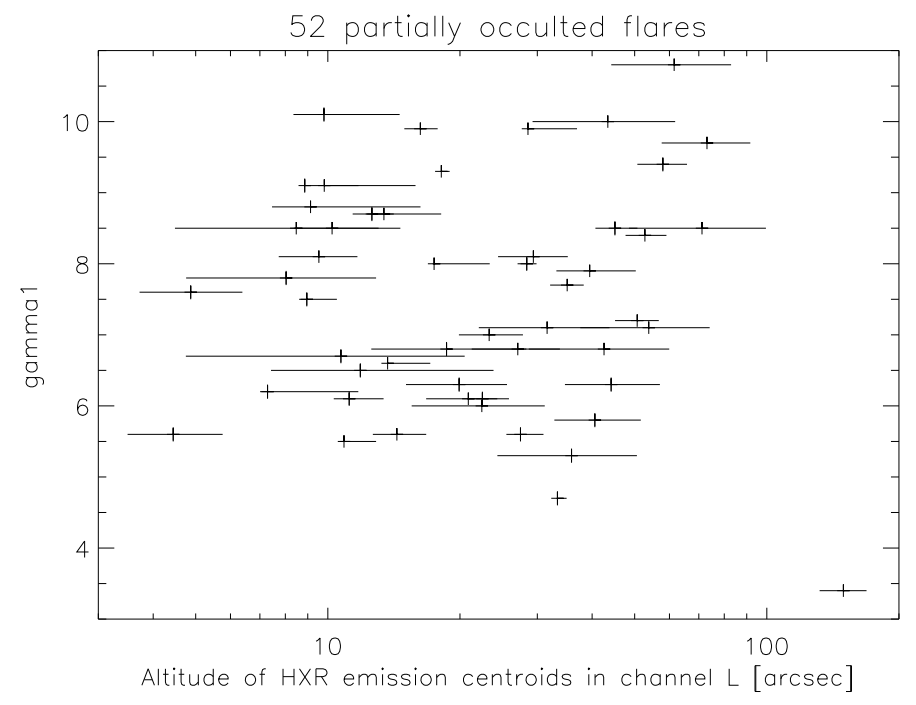

Fig. 9. The power-law index $\gamma 1$ measured between $14-33 \mathrm{keV}$ against the altitude of the centroid of hard X-ray emission imaged in energy band $\mathrm{L}$ for 52 partially occulted flares.

free-bound to a free-free dominated spectrum was suggested by Brown \& Mallik (2008).

\subsection{Location of coronal hard X-ray sources}

We use the imaging ability of HXT to investigate the spatial structure of the coronal sources. In the first step, we checked the dependence of observed hard X-ray spectra on the altitude of coronal sources above the solar surface.

In Fig. 9, we compared the power-law index $\gamma 1$ to the altitude of the centroid of hard X-ray emission imaged in energy band L for 52 partially occulted flares. In the case of multiple sources, the location of the brightest one was considered. The altitude was the sum of two components of a height above the solar limb $h_{1}$ (Col. 9 in Table 2) and a occultation height $h_{2}$. The latter one was calculated on the basis of heliospheric coordinates (Col. 6 in Table 2) to be:

$h_{2}=[\sec \theta-1] \sec \psi R_{\odot}$,

where $\theta$ is the longitude behind the solar limb and $\psi$ is the latitude. The main source of the altitude error is the uncertainty in the longitude (Col. 7 in Table 2). As we can see, no correlation is observed - flares exhibiting hard X-ray spectra of different shapes occur at each altitude. We also found a lack of correlation between the altitude and the power-law index for higher energy ranges.

How are the non-thermal and thermal components in investigated flares spatially located? To find out, we compared the location of coronal hard X-ray sources to the location of bright loop-top kernels seen in soft X-rays. In Fig. 10, we compared a shift between centroids of emission in these two ranges of X-rays with the altitude of the flares. We considered images of 41 partially occulted flares for which the hard X-ray image in energy band L and the soft X-ray image taken in the Be119 filter were obtained simultaneously. For clarity, error bars are omitted. The typical error in the shift was estimated to be about 2 arcsec. The altitude error is the same as in Fig. 9.

For 26 events $(63.4 \%)$, the separation between the nonthermal and thermal components is not higher than 5 arcsec. The maximal values of the shift seem to increase with altitude. 


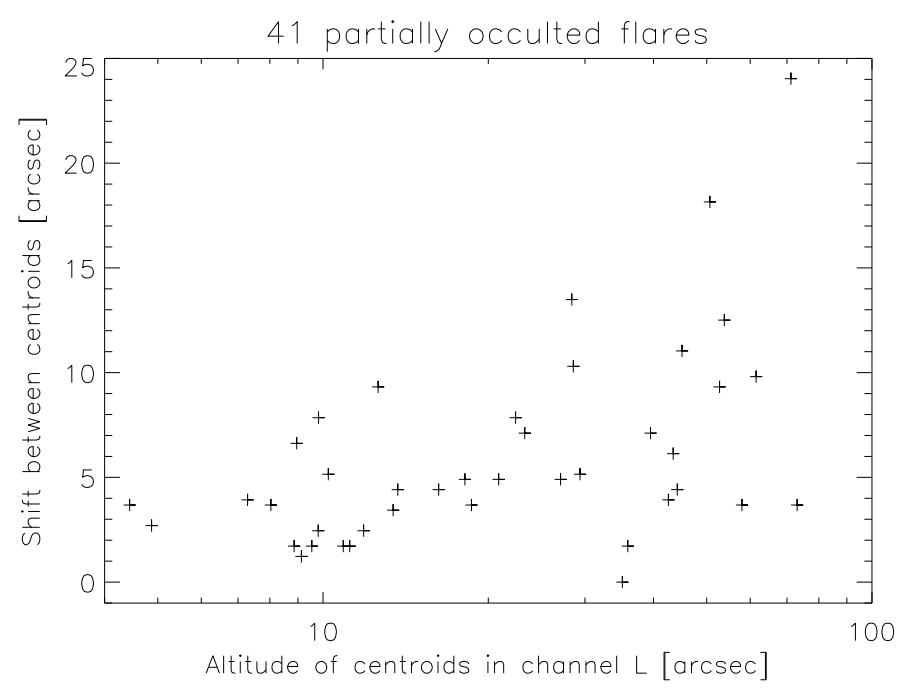

Fig. 10. The shift between centroids of hard (HXT, energy band L) and soft (SXT, Be119 filter) X-ray emission against the altitude of the centroid of hard X-ray emission for 41 partially occulted flares.

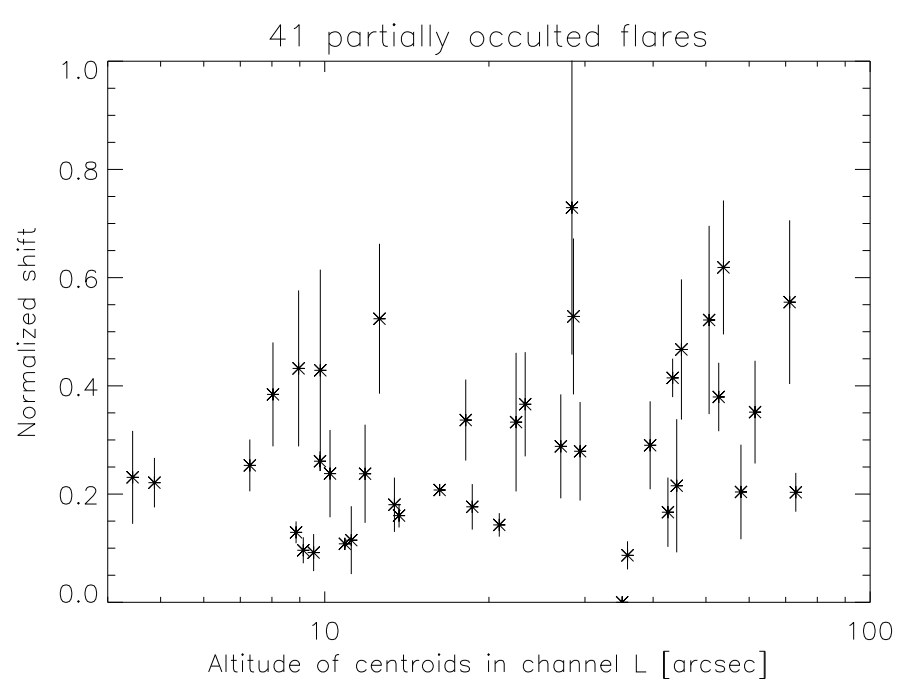

Fig. 11. Shifts from Fig. 10 normalized by dividing with characteristic sizes of hard X-ray sources in energy band $\mathrm{L}$ against the altitude of flares for 41 partially occulted flares. For further explanations - see text.

However, this appearance can be caused by an increase with altitude in magnetic features sizes, e.g., soft X-ray loop-top kernels (Preś \& Kołomański 2007). To check, we normalized the values presented in Fig. 10 by dividing the shift by the characteristic size of a hard X-ray source.

We calculated the range of values of the normalized shift seen in Fig. 11 as follows. We approximated the hard X-ray sources at the level of $0.5 I_{\max }$ as ellipses and divided the shifts from Fig. 10 by the large and small semi-axis of the ellipse. It establishes a lower and higher boundary of the normalized shift, respectively. As we can see, the normalized shift does not depend on altitude, and for the majority of events (36 from 41) does not exceed 0.5 . The true cospatiality of hard X-ray and soft X-ray emission for some investigated events is even better because small semi-axes (high boundaries of normalized shifts) were shortened due to an occultation of the solar limb.

Figures 10 and 11 do not distinguish which kind of emission, soft or hard X-ray, is located higher. On the other hand, this appearance is crucial for fitting the data with some theoretical

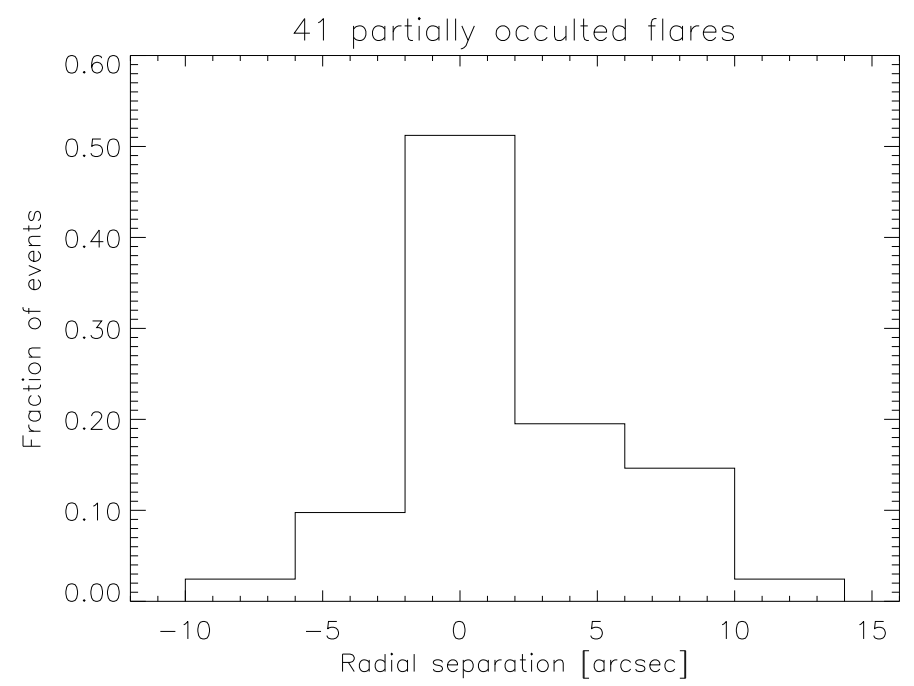

Fig. 12. The radial separation between centroids of hard X-ray and soft $\mathrm{X}$-ray emission for 41 partially occulted flares from Fig. 10. In the most prominent example, the Masuda flare, the radial separation was about 10 arcsec.

models. Therefore, in Fig. 12 we present the radial separation between the centroids of hard X-ray and soft X-ray emission for 41 partially occulted flares from Fig. 10. Almost half of these events (21 from 41) are of the same altitude for both hard X-ay and soft $\mathrm{X}$-ray emission, to within error bars. The second half show differences between them of up to 14 arcsec with a supremacy of events in which hard X-ray emission is located higher than soft $\mathrm{X}$-ray emission ( 15 from remaining 20 ). For 5 events, soft X-ray emission was located higher than hard X-ray emission.

In Figs. 9-12, we used images taken in the energy band $\mathrm{L}$ as a representation of hard X-ray emission of investigated flares. One may doubt conclusions based on observations in this energy band because of the strong contamination by emission of thermal plasma. Perhaps, Figs. 11 and 12 imply that the hard and soft X-ray emission are cospatial because we observe the same thermal plasma? To verify this idea, we compare the location of the centroids of emission in energy band $\mathrm{L}$ to those imaged of higher energies. Unfortunately, a smaller number of events could be checked in this way, because for energy bands M1 and M2, only 16 and 5 images were available, respectively. For only two events, Nos. 11950 and 25870 , we found that the radial separation between emission in energy band $\mathrm{L}$ and at higher energies is greater than 4 arcsec. The remaining 14 events are almost perfectly cospatial.

\section{Discussion and interpretation of results}

Table 5 collects the results of surveys in which hard X-ray spectra of loop-top sources were investigated and compared to spectra of footpoint sources. The large number of solar flares included in these surveys implies that the results should be statistically important.

Table 5 contains two kinds of data. Papers (1), (2), (4), and (6) described a set of partially occulted flares and presented spectra of coronal hard X-ray sources. Apart from Paper (6), these papers also presented, for a comparison, spectra of non-occulted flares. They are a mixture of emission of loop-top and footpoint sources, although their spectra are understood to be dominated by footpoint sources, especially at higher energies. Based on the imaging spectroscopy, Papers (3) and (5) provide the 
Table 5. Surveys comparing power-law spectrum indices of hard X-ray loop-top and footpoint sources.

\begin{tabular}{|c|c|c|c|c|c|c|c|c|c|c|c|c|c|c|c|c|}
\hline \multirow{3}{*}{ Satellite } & \multirow{3}{*}{$\begin{array}{c}\text { Time } \\
\text { period } \\
{[\mathrm{YY} / \mathrm{MM}]}\end{array}$} & \multirow{3}{*}{$\begin{array}{l}\text { Energy } \\
\text { range } \\
{[\mathrm{keV}]} \\
\end{array}$} & \multirow{3}{*}{$\begin{array}{c}\text { Energy } \\
\text { res. } \\
{[\mathrm{keV}]}\end{array}$} & \multicolumn{12}{|c|}{ Power-law index $\gamma^{a}$} & \multirow{3}{*}{ Refs. $^{b}$} \\
\hline & & & & \multicolumn{6}{|c|}{ loop-top } & \multicolumn{6}{|c|}{ footpoint } & \\
\hline & & & & $\mathrm{N}$ & $\overline{M n}$ & $\overline{\mathrm{Md}}$ & SD & $\bar{K}$ & $\mathrm{~S}$ & $\mathrm{~N}$ & $\mathrm{Mn}$ & Md & SD & $\bar{K}$ & $\mathrm{~S}$ & \\
\hline$O S O-7$ & $71 / 10-72 / 12$ & $10-300$ & $\sim 30$ & 25 & 4.8 & 4.8 & 1.0 & -1.0 & 0.1 & 59 & 3.9 & 3.8 & 1.1 & -0.6 & 0.6 & (1) \\
\hline Yohkoh & $91 / 10-96 / 05$ & $14-33$ & 10 & 25 & 8.2 & 8.1 & 1.5 & -1.1 & -0.1 & 16 & 7.3 & 7.7 & 1.3 & -0.9 & -0.4 & (2) \\
\hline Yohkoh & $91 / 10-98 / 08$ & $14-53$ & $10-20$ & $12^{c}$ & 6.6 & 6.2 & 1.5 & 0.2 & 0.4 & $18^{c}$ & 5.2 & 4.9 & 1.6 & -1.0 & 0.4 & (3) \\
\hline Yohkoh & $91 / 10-98 / 08$ & $14-93$ & $10-40$ & $5^{c}$ & 5.5 & 5.3 & 1.3 & -1.5 & 0.2 & $13^{c}$ & 4.3 & 4.1 & 1.0 & -1.0 & 0.7 & (3) \\
\hline Yohkoh & $91 / 10-01 / 12$ & $14-33$ & 10 & 52 & 7.5 & 7.6 & 1.6 & -0.5 & -0.1 & 620 & 4.8 & 4.6 & 1.6 & -0.6 & 0.4 & (4) \\
\hline Yohkoh & $91 / 10-01 / 12$ & $23-53$ & 10-20 & 23 & 6.7 & 6.9 & 1.4 & -0.7 & -0.2 & 338 & 4.0 & 3.8 & 1.1 & 2.1 & 1.0 & (4) \\
\hline Yohkoh & $91 / 10-01 / 12$ & $33-93$ & $20-40$ & 3 & - & - & - & - & - & 155 & 3.4 & 3.4 & 0.7 & 1.2 & 0.6 & (4) \\
\hline Yohkoh & $91 / 10-01 / 12$ & $14-53$ & $10-20$ & $7^{d}$ & 5.6 & 5.2 & 1.4 & -1.3 & 0.2 & $185^{d}$ & 3.9 & 3.7 & 1.1 & 0.8 & 0.8 & (4) \\
\hline Yohkoh & $91 / 10-01 / 12$ & $23-93$ & $10-40$ & $1^{d}$ & - & - & - & - & - & $130^{d}$ & 3.3 & 3.2 & 0.7 & 1.4 & 0.6 & (4) \\
\hline RHESSI & $02 / 02-05 / 07$ & $3-1.7 \times 10^{4}$ & $1-5$ & $5^{c}$ & 5.1 & 5.6 & 1.2 & -2.0 & -0.3 & $10^{c}$ & 2.9 & 2.8 & 0.5 & -1.4 & 0.1 & (5) \\
\hline RHESSI & $02 / 02-04 / 08$ & $3-1.7 \times 10^{4}$ & $1-5$ & 50 & 5.4 & 5.5 & 1.2 & -0.8 & 0.3 & 0 & - & - & - & - & - & (6) \\
\hline RHESSI & $02 / 02-05 / 08$ & $3-1.7 \times 10^{4}$ & $1-5$ & 0 & - & - & - & - & - & $174^{c}$ & 3.3 & 3.1 & 0.5 & 0.3 & 0.7 & (7) \\
\hline
\end{tabular}

${ }^{a} \mathrm{~N}$ - number of events; Mn - mean, Md - median, SD - standard deviation, K - kurtosis; S - skewness.

${ }^{b}$ (1) - Roy \& Datlowe (1975); (2) - Mariska \& McTiernan (1999); (3) - Petrosian et al. (2002); (4) - this issue; (5) - Battaglia \& Benz (2006, 2007); (6) - Krucker \& Lin (2008); (7) - Saint-Hilaire et al. (2008).

${ }^{c}$ Imaging spectroscopy.

${ }^{d}$ Events unbiased by the thermal component.

characteristics of the spectra of loop-top and footpoint sources of the same flares. Paper (7) also used the imaging spectroscopy but was limited to footpoint sources.

Histograms of values of the power-law index are represented in Table 5 by routine statistical parameters: mean, median, standard deviation, kurtosis, and skewness. In Papers (1) and (7), the source data were not published, therefore their statistical parameters were calculated based on the assumption that all events in a particular bin equaled to the middle value. The number of events relates to the number of flares or the number of hard X-ray sources in imaging spectroscopy. For Yohkoh observations, the energy ranges of spectral fitting are given. In the case of other observations basically, the full available energy coverage were used, in exception to Paper (7) in which the power-law was fitted above the cut-off energy.

The histograms gathered in Table 5 exhibit different shapes for loop-top and footpoint sources. On the other hand, the histograms obtained for the same group of sources show similar shapes. Distributions of spectra of the loop-top sources show basically broad maxima, which are almost symmetrical with a small excess for flatter spectra. Distributions of spectra of the footpoint sources show generally narrower maxima and are strongly asymmetric with an excess for steeper spectra.

To describe the most characteristic values of $\gamma$, we use medians, because of the large skewness of distributions, especially for footpoint sources. The broad range of medians within both groups of sources is seen. This wide spread can be caused by many factors such as instrument properties, methods of spectral selection and fitting, or criteria of selection of flares. Nevertheless, all surveys performed have shown that hard X-ray spectra of loop-top sources are systematically steeper than spectra of footpoint sources.

The medians obtained from low-energy bands of the HXT are in particular shifted towards the higher values, due to strong contamination by the thermal component. In Paper (2), very long times of integration additionally favored the influence of the thermal component. To avoid this contamination, it is recommended that we shift the spectral fitting towards higher energies, although this activity limits the number of considered events and attributes the results with a selection effect. The comparison of slopes in different energy ranges of the hard X-ray spectrum, performed in Figs. 6 and 7, allowed us to isolate some flares not produced by the thermal component even at lower energies. Because of this we were able to improve the quality of our statistical analysis and our results look reasonable in comparison with medians obtained from surveys other than Yohkoh.

Medians of histograms from Papers (1), (5), (6), and (7) are within the ranges of 4.7-5.5 and 3.1-3.8 for loop-top and footpoint sources, respectively. However, even these values are not completely free of systematic effects. The spectrum of the nonocculted flare is a mixture of photons emitted from both footpoints and the corona. Despite an usual predominance of footpoints, the use of the spectrum of the non-occulted flare as a representation of footpoint sources introduces some bias. On the other hand, imaging spectroscopy can distort spectra of fainter sources (usually the loop-top ones) in the presence of brighter sources (usually the footpoint ones). To overcome this limit, partially occulted flares are chosen, although imprecise selection can be confusing. This was probably true for Paper (1) in which limb flares $\left(60^{\circ}<\theta<90^{\circ}\right)$ exhibited similar values of $\gamma$ to those obtained for partially occulted flares. In Paper (6), the distribution at high $\gamma$ is distorted by observational bias because only flares with hard spectra (above $50 \mathrm{keV}$ ) were studied.

We confirmed the presence of a non-thermal component in 11 of the $23(\sim 48 \%)$ partially occulted flares. In 10 of the 23 flares $(\sim 43 \%)$, some impulsive non-thermal episodes similar to those described by Tomczak (2001) were seen, but the impulses modified the thermal appearance of these flares only slightly. In earlier surveys, the non-thermal component was discovered in 25 of 37 (Roy \& Datlowe 1975) and in 50 of 55 flares (Krucker \& Lin 2008), i.e., in $\sim 68 \%$ and $\sim 91 \%$ of events, respectively. We also estimated the frequency of the occurrence of nonthermal components in loop-top sources described by Petrosian et al. (2002), by comparing values of $\gamma$ in $14-34$ and $23-53 \mathrm{keV}$ energy ranges. The result was $\sim 65 \%$ (11 of 17 events).

The spatial location of the non-thermal and thermal components is common for the majority of investigated flares. The same rule can be concluded from the earlier papers (Tomczak 2001; Krucker \& Lin 2008). Masuda (1994) found that the coronal hard X-ray sources located above the soft X-ray loop-top kernels show spectra that are as flat as the spectra of the footpoint sources. Alexander \& Metcalf (1997) obtained a higher 
value, $\gamma_{\mathrm{LT}} \sim 4$, which implies a distinctly flatter spectrum than for a typical coronal source. In our survey, flares, for which the hard X-ray and soft X-ray components are spatially separated, do not have unusually flat spectra. The extremely shifted event in the survey of Krucker \& Lin (2008), i.e., the flare of 2003 November 18, also exhibits a modest value of $\gamma$ equal to 5.6.

What do the spectra of hard X-ray sources teach us about non-thermal electron beams in solar flares? In the simplest scenario, the same electron beam, described by the power-law in$\operatorname{dex} \delta$, radiates hard X-ray photons in the thin-target loop-top and thick-target footpoint sources. Spectra emitted by these two kinds of sources have indices that differ by 2: $\gamma_{\mathrm{LT}}=\delta+1$ and $\gamma_{\mathrm{FP}}=\delta-1$. Mariska et al. (1996) measured such a difference and presented the interpretation of partially occulted and nonocculted flares, but this small number of investigated events (4+ 4) requires verification by additional data.

Without exception, the surveys presented in Table 5 confirm that the loop-top sources exhibit spectra that are systematically steeper than those of the footpoints, i.e., $\bar{\gamma}_{\mathrm{LT}}>\bar{\gamma}_{\mathrm{FP}}$. In this paper, we measured the differences between the medians of the distributions of $\gamma$ values, $\bar{\gamma}_{\mathrm{LT}}-\bar{\gamma}_{\mathrm{FP}}$, to be about 3 . However, the results are affected significantly by contamination from the thermal component. The difference, $\Delta \gamma=\bar{\gamma}_{\mathrm{LT}}-\bar{\gamma}_{\mathrm{FP}}$, for unaffected events is about 1.5.

The difference, $\Delta \gamma<2$, means that part of the investigated flares have properties that do not agree with the simplest scenario, $\Delta \gamma=2$. The shape of the distribution of $\gamma$ values, which is more symmetric and broader for loop-top sources than footpoint ones, can provide a solution. We guess that some looptop sources that emitted hard X-rays in the thick-target mechanism are responsible for this. A low-value wing of the $\gamma$ distribution for loop-top sources is occupied by flares in which $\mathrm{X}$-ray plasma ejections were observed, hence perhaps a kind of magnetic trapping occurs. Another opportunity for coronal thick-target emission may be an unusually-high filling of a flare loop with plasma (Kosugi et al. 1994; Veronig \& Brown 2004).

Values of the difference $\Delta \gamma$ higher than 2, obtained by analyses of RHESSI observations (Battaglia \& Benz 2006, 2007; Krucker \& Lin 2008; Saint-Hilaire et al. 2008), require other explanations. For example, separate non-thermal electron beams responsible for loop-top and footpoint sources, deceleration of the precipitating electrons in electric fields due to return currents (Battaglia \& Benz 2008), or non-thermal recombination (Brown $\&$ Mallik 2008) should be instead be considered.

A lack of correlation between the altitude and the power-law index $\gamma$, seen in Fig. 9, suggests that the investigated flares do not respond to the overall (global) magnetic configuration of the solar corona. Their characteristics clearly imply the local magnetic configuration in which these flares were developed.

We identified a non-thermal component in a majority of the investigated flares, in a similar way to the earlier surveys. We have pointed out that in most cases the non-thermal and thermal components (the hard X-ray loop-top source and the bright loop-top kernel, respectively) are cospatial or overlap during the impulsive phase, which confirms that both types of emission come from the common plasma volume and are strongly coupled. Events in which both components are clearly separated, such as a "Masuda flare", seldom occur and are not obviously characterized by a flat hard X-ray spectrum. A similar conclusion was reached by Krucker \& Lin (2008).

We emphasize the clear inconsistency between a picture of a flare that is shaped by statistical surveys (i.e., what their typical properties are) and by individual famous events. On the other hand, the models adopted to explain a specific configuration, such as a Masuda flare, are used often as a kind of unification, i.e., explanation of all flares. There is no doubt that their frequency of occurrence should not be a conclusive criterion for performing an interpretation, but there is no reason to recognize such unusual structures, such as a Masuda flare, in each flare, as well.

Why are Masuda-type flares poorly represented in statistical surveys such as ours, even though new examples are more recently reported (Shimizu et al. 2008)? Such an unusual appearance is seen during short episodes occurring randomly in time. On the other hand, one flare is usually represented in catalogues by one time period around the maximum. Thus, it is quite easy to miss such an interesting episode. For example, the famous above-the-loop-top hard X-ray source discovered in a Masuda flare is seen at the beginning of the impulsive phase, and during the hard X-ray maximum it is dominated by the strong footpoint sources. In conclusion, searching for interesting episodes needs careful selection of a sequence of images illustrating the evolution.

\section{Interesting groups of events}

We provide short descriptions of interesting group of events from our survey.

\subsection{Progressive spectral hardening}

A relatively less frequent group of flares shows in hard X-rays a progressively hardening spectral evolution that can be described schematically by the pattern "soft-hard-harder" (SHH). These flares are interesting because of their association with other solar activity phenomena such as Coronal Mass Ejections, Solar Energetic Particles, radio bursts type II and type IV. Early reports located the radiation of the SHH flares clearly above the solar surface, inside a postflare loop system where electrons are accelerated and trapped (Cliver et al. 1986, and references therein). However, this picture was directly confirmed only for a few events that occurred some distance behind the solar limb or were imaged below $40 \mathrm{keV}$ by Hinotori.

The Yohkoh HXT introduced a new picture of SHH flares in which their emission originates close to the solar surface, in flare footpoints (Qiu et al. 2004; Takasaki et al. 2007). The dominance of footpoint emission was also confirmed for some $\mathrm{SHH}$ flares observed by RHESSI (Saldanha et al. 2008; Grigis \& Benz 2008). Thick-target footpoint sources primarily emit their hard X-rays immediately, and the SHH spectral pattern cannot therefore be explained by the trapping mechanism alone. Footpoint SHH flares strongly imply a physical mechanism that continuously accelerates electrons to ever higher energies is involved.

In our survey, we have found three flares exhibiting the $\mathrm{SHH}$ spectral pattern. Their basic characteristics are given in Table 6 . All occurred far enough behind the solar limb to eliminate the hard X-ray emission from footpoints, and because of this, closer insight into the corona was possible. Tomczak (2008) noted that the SHH spectral pattern coincides in time with the occurrence of a new coronal source. In the case of 1998 May 9, this source moved away gradually, its velocity increasing from 25 to $80 \mathrm{~km} \mathrm{~s}^{-1}$.

The progressive spectral hardening in flares from Table 6 was always preceded by a phase of the "soft-hard-harder" (SHH) spectral evolution pattern in which another hard X-ray source was seen. This behavior strongly suggests that a switch between the SHS and SHH spectral pattern required significant reconfiguration of the magnetic structure of a flare. 
Table 6. List of flares showing progressive spectral hardening.

\begin{tabular}{clccccc}
\hline \hline $\begin{array}{c}\text { Catalogue } \\
\text { number }\end{array}$ & Date & $\begin{array}{c}\text { GOES } \\
\text { class }\end{array}$ & Longitude & $\begin{array}{c}\text { Occultation } \\
\text { height }[\mathrm{Mm}]\end{array}$ & $\gamma$ range & $\begin{array}{c}\text { Time } \\
\text { interval }\end{array}$ \\
\hline 9700 & 1994 February 27 & M2.8 & W97.5 & 6.1 & $6 \rightarrow 4$ & $09: 09-09: 18$ \\
11950 & 1998 May 9 & M7.7 & W102.3 & 17.1 & $6.0 \rightarrow 3.4$ & $03: 29-03: 39$ \\
$25540^{a}$ & 2001 April 1 & M5.5 & E107.0 & 34.1 & $>11 \rightarrow 7.6^{b}$ & $11: 48-11: 54$ \\
\hline
\end{tabular}

${ }^{a}$ Only the decay phase is available.

${ }^{b}$ Strong contamination with emission of thermal plasma.

Table 7. List of Yohkoh coronal $\gamma$-ray flares.

\begin{tabular}{clcccc}
\hline $\begin{array}{c}\text { Catalogue } \\
\text { number }\end{array}$ & Date & $\begin{array}{c}\text { GOES } \\
\text { class }\end{array}$ & Longitude & $\begin{array}{c}\text { Occultation } \\
\text { height }[\mathrm{Mm}]\end{array}$ & $\begin{array}{c}\text { Photons } \\
\text { energy }[\mathrm{keV}]\end{array}$ \\
\hline 6240 & 1992 November 2 & X9.0 & W97.9 & 7.4 & $<600^{a}$ \\
11650 & 1998 April 23 & X1.2 & E103.7 & 21.4 & $<250$ \\
11950 & 1998 May 9 & M7.7 & W102.3 & 17.1 & $<200$ \\
13650 & 1998 November 24 & X1.0 & W103.1 & 21.5 & $<600^{a}$ \\
26060 & 2001 April 18 & C2.2 & W116.9 & 90.5 & $<250$ \\
\hline
\end{tabular}

${ }^{a}$ Only the decay phase is available.

\subsection{Coronal $\gamma$-ray sources}

Because of the RHESSI imaging capabilities it is possible to obtain hard X-ray images at higher energies than possible before. The results, which are limited by counting statistics and dynamic range, show that $\gamma$-rays are usually emitted from footpoints (Saldanha et al. 2008), although an example of the coronal source is also known (Krucker et al. 2008b). Yohkoh could not image at such high energies, although in the HXT Flare Catalogue we found five events, recorded by the WBS, that occurred behind the solar limb and for which spectra reach at least $200 \mathrm{keV}$ (see Table 7).

All these events occurred far enough behind the solar limb to be certain that the high-energy photons are emitted from the corona. In this way, without imaging, we obtain an independent confirmation that $\gamma$-rays can be produced in the corona. The flare of 1998 November 24, which produced 600-keV photons about 3 minutes after its maximum, attracted special attention. According to the CGRO/BATSE light curve, the flux decreased almost twice during this time interval, hence photons more energetic than $600 \mathrm{keV}$ at most were emitted.

Krucker et al. (2008b) interpreted the coronal $\gamma$-ray emission as relativistic electron-electron bremsstrahlung at energies perhaps of a few MeV. Therefore, these observations directly imply that flare-accelerated $\mathrm{MeV}$ electrons reside stably in the corona, losing their energy collisionally and producing $\gamma$-ray continuum.

\section{Conclusions}

Among the 1286 flares detected in the Yohkoh Hard X-ray Telescope Flare Catalogue, for which hard X-ray images had been enclosed, we identified 98 events that occurred behind the solar limb. The obscurity of footpoints, which are usually brighter in hard X-rays, allowed us to isolate the coronal parts of these flares for a more detailed analysis. We investigated hard $\mathrm{X}$-ray spectra and the spatial structure of partially occulted flares. In most cases, we found that their hard X-ray spectra consists of two cospatial components, non-thermal and thermal.

We note that the spectra of partially occulted flares are systematically steeper than spectra of non-occulted events in the catalogue. This shows that the hard X-ray emission in solar flares is usually less energetic in the corona (loop-top sources) than close to the solar surface (footpoint sources). The difference between the median values of the power-law index for both classes of sources, $\Delta \gamma=\bar{\gamma}_{\mathrm{LT}}-\bar{\gamma}_{\mathrm{FP}}$, is about 3 . However, this result is strongly affected by thermal emission. For events unbiased by the thermal component, the difference $\Delta \gamma$ equals 1.5. The value obtained is similar to the results of other surveys.

We conclude that the different slopes of the non-thermal component are basically caused by different emission mechanisms: thin-target for loop-top sources and thick-target for footpoint sources. The difference of $\Delta \gamma$, which is smaller than 2 , suggests that exceptions to this rule exist. We have presented arguments that part of coronal hard X-ray sources is a thick-target instead of a thin-target, e.g., several unusually high-energetic events that occurred in the configuration indicative of magnetic trapping. An additional argument that flare characteristics are dependent on the local magnetic configuration in which they develop, reflects a lack of correlation between the altitude of flares and the hard X-ray power-law index $\gamma$.

Additional high-quality observations and high-precision analyses are needed to derive further details about non-thermal electron acceleration and propagation in solar flares. Detailed imaging spectroscopy of individual events as well as massive surveys of many flares are desired.

Acknowledgements. The Yohkoh satellite is a project of the Institute of Space and Astronautical Science of Japan. I am grateful to late Professor Takeo Kosugi, Dr. Jun Sato, and their collaborators for preparing the Yohkoh HXT Flare Catalogue which made this work easier. I thank also my colleagues from a working group of the International Space Science Institute (Bern, Switzerland), dedicated to coronal hard X-ray sources, for a fruitful discussion. I appreciate valuable remarks of referee, Dr. S. Krucker, which helped to improve this paper. This work was supported by Polish Ministry of Science and High Education grant No. N N203 193733.

\section{References}

Alexander, D., \& Metcalf, T. R. 1997, ApJ, 456, 751 Battaglia, M., \& Benz, A. O. 2006, A\&A, 456, 751 Battaglia, M., \& Benz, A. O. 2007, A\&A, 466, 713 Battaglia, M., \& Benz, A. O. 2008, A\&A, 487, 337 Brown, J. C. 1971, Sol. Phys., 18, 489

Brown, J. C., \& Mallik, P. C. V. 2008, A\&A, 481, 507 
Catalano, C. P., \& Van Allen, J. A. 1973, ApJ, 185, 335

Cliver, E. W., Dennis, B. R., Kiplinger, A. L., et al. 1986, ApJ, 305, 920

Frost, K. J., \& Dennis, B. R. 1971, ApJ, 165, 655

Grigis, P. C., \& Benz, A. O. 2008, ApJ, 683, 1180

Hudson, H. S. 1978, ApJ, 224, 235

Hudson, H. S., Lin, R. P., \& Stewart, R. T. 1982, Sol. Phys., 75, 245

Hudson, H. S., Strong, K. T., Dennis, B. R., et al. 1994, ApJ, 422, L25

Hudson, H. S., Kosugi, T., Nitta, N. V., \& Shimojo, M. 2001, ApJ, 561, L211

Kane, S. R., Fenimore, E. E., Klebesadel, R. W., \& Laros, R. G. 1982, ApJ, 254, L53

Kašparova, J., Kontar, E. P., \& Brown, J. C. 2007, A\&A, 466, 705

Khan, J. I., Harra-Murnion, L. K., Hudson, H. S., Lemen, J. R., \& Sterling, A. C. 1995, ApJ, 452, L153

Kosugi, T., Makishima, K., Murakami, T., et al. 1991, Sol. Phys., 136, 17

Kosugi, T., \& Yohkoh HXT Group 1993, The Yohkoh HXT Databook (I), 1991 October-1992 December, National Astronomical Observatory, Mitaka, Tokyo, Japan (Version 1)

Kosugi, T., Sakao, T., Masuda, S., et al. 1994, Proc. Kofu Symposium: New Look at the Sun with Emphasis on Advanced Observations of Coronal Dynamics and Flares, ed. S. Enome, \& T. Hirayama, Nobeyama Radio Observatory Report No. 360, 127

Kosugi, T., Sawa, M., Sakao, T., et al. 1995, The Yohkoh HXT Databook: October 1991-December 1994, National Astronomical Observatory, Mitaka, Tokyo, Japan (Version 2)

Krucker, S., \& Lin, R. P. 2008, ApJ, 673, 1181

Krucker, S., White, S. M., \& Lin, R. P. 2007a, ApJ, 669, L49

Krucker, S., Hannah, I. G., \& Lin, R. P. 2007b, ApJ, 671, L193

Krucker, S., Battaglia, M., Cargill, P. J., et al. 2008a, A\&ARv, 16, 155

Krucker, S., Hurford, G. J., McKinnon, A. L., Shih, A. Y., \& Lin, R. P. 2008b, ApJ, 678, L63

Krucker, S., Hudson, H. S., White, S. M., Wuelser, J.-P., \& Lin, R. P. 2009, in preparation

Li, Y.P., \& Gan, W. Q. 2006, ApJ, 652, L61

Li, Y. P., \& Gan, W. Q. 2007, Adv. Space Res., 39, 1389

Lin, R. P. 1974, Space Sci. Rev., 16, 189

Liu, W., Petrosian, V., Dennis, B. R., \& Jiang, Y. W. 2008, ApJ, 676, 704

Mariska, J. T., \& Doschek, G. A. 1997, ApJ, 485, 904
Mariska, J. T., \& McTiernan, J. M. 1999, ApJ, 514, 484

Mariska, J. T., Sakao, T., \& Bentley, R. D. 1996, ApJ, 459, 815

Masuda, S. 1994, Ph.D. Thesis, University of Tokyo

Masuda S., Kosugi, T., Hara, H., Tsuneta, S., \& Ogawara, Y. 1994, Nature, 371, 495

McKenzie, D. L. 1975, Sol. Phys., 40, 183

Newton, H. W., \& Nunn, M. L. 1951, MNRAS, 111, 413

Ohyama, M., \& Shibata, K. 1997, PASJ, 49, 249

Petrosian, V., Donaghy, T. Q., \& McTiernan, J. M. 2002, ApJ, 569, 459

Preś, P., \& Kołomański, S. 2007, Cent. Eur. Astrophys. Bull., 31, 87

Qiu, J., Lee, J., \& Gary, D. E. 2004, ApJ, 603, 335

Roy, J.-R., \& Datlowe, D. W. 1975, Sol. Phys., 40, 165

Sato, J. 2001, ApJ, 558, L137

Sato, J., Sawa, M., Masuda, S., et al. 1998, The Yohkoh HXT Image Catalogue: October 1991-August 1998, Nobeyama Radio Observatory, National Astronomical Observatory, Japan (Version 3)

Sato, J., Sawa, M., Yoshimura, K., Masuda, S., \& Kosugi, T. 2003, The Yohkoh HXT/SXT Flare Catalogue, Montana State University and Institute of Space and Astronautical Science (Version 4)

Sato, J., Matsumoto, Y., Yoshimura, K., et al. 2006, Sol. Phys., 236, 351 (Version 5)

Saint-Hilaire, P., Krucker, S., \& Lin, R. P. 2008, Sol. Phys., 250, 53

Saldanha, R., Krucker, S., \& Lin, R. P. 2008 ApJ, 673, 1169

Shimizu, M., Nishida, K., Takasaki, H., et. al. 2008, ApJ, 683, L203

Sterling, A. C., Harra-Murnion, L. K., Hudson, H. S., \& Lemen, J. R. 1996, ApJ, 464, 498

Takasaki, H., Kiyohara, J., Asai, A., et al. 2007, ApJ, 661, 1234

Tomczak, M. 1997, A\&A, 317, 223

Tomczak, M. 2001, A\&A, 366, 294

Tomczak, M. 2004, A\&A, 417, 1133

Tomczak, M. 2005, Adv. Space Res., 35, 1732

Tomczak, M. 2008, Cent. Eur. Astrophys. Bull., 32, 59

Tomczak, M., \& Ciborski, T. 2007, A\&A, 461, 315

Veronig, A. M., \& Brown, J. C. 2004, ApJ, 603, L117

Ward, F. 1966, ApJ, 145, 416

Zhang, J., \& Huang, G. L. 2003, ApJ, 592, L49

Zirin, H., Ingham, W., Hudson, H., \& McKenzie, D. 1969, Sol. Phys., 9, 269 